Pure and Applied Mathematics Quarterly

Volume 3, Number 1

(Special Issue: In honor of

Robert MacPherson, Part 3 of 3)

$25-60,2007$

\title{
Moment-angle Complexes, Monomial Ideals and Massey Products
}

\author{
Graham Denham ${ }^{1}$ and Alexander I. Suciu ${ }^{2}$ \\ To Robert MacPherson on the occasion of his sixtieth birthday
}

\begin{abstract}
Associated to every finite simplicial complex $K$ there is a "moment-angle" finite $\mathrm{CW}$-complex, $\mathcal{Z}_{K}$; if $K$ is a triangulation of a sphere, $\mathcal{Z}_{K}$ is a smooth, compact manifold. Building on work of Buchstaber, Panov, and Baskakov, we study the cohomology ring, the homotopy groups, and the triple Massey products of a moment-angle complex, relating these topological invariants to the algebraic combinatorics of the underlying simplicial complex. Applications to the study of non-formal manifolds and subspace arrangements are given.
\end{abstract}

\section{Contents}

1. Introduction 26

2. The moment-angle functor 29

3. Cohomology and the Stanley-Reisner ring 34

4. Homotopy groups and Koszul algebras 37

5. The cellular cochain algebra and Massey products 39

6. Triple Massey products in lowest degree 44

Received December 7, 2005.

2000 Mathematics Subject Classification. Primary 13F55, 55S30 Secondary 16E05, 32Q55, 55P62, 57R19.

key words and phrases. moment-angle complex, cohomology ring, homotopy Lie algebra, Stanley-Reisner ring, Taylor resolution, Eilenberg-Moore spectral sequence, cellular cochain algebra, formality, Massey product, triangulation, Bier sphere, subspace arrangement, complex manifold.

${ }^{1}$ Partially supported by a grant from NSERC of Canada.

${ }^{2}$ Partially supported by NSF grant DMS-0311142. 
7. Formal moment-angle manifolds $\quad 47$

8. Non-formal moment-angle manifolds 50

9. Subspace arrangements $\quad 55$

$\begin{array}{ll}\text { References } & 58\end{array}$

\section{INTRODUCTION}

1.1. Moment-angle complexes. A construction due to Davis and Januszkiewicz [12] and studied in detail by Buchstaber and Panov [9] associates to every simplicial complex $K$ on $n$ vertices a finite cellular complex $\mathcal{Z}_{K}$, endowed with a natural action by the $n$-torus, and whose orbit space is the cone over $K$.

The unit disk $D^{2} \subset \mathbb{C}$ has a natural cell structure, in which the boundary circle $S^{1}$ is the 1 -skeleton. The $n$-polydisk $\left(D^{2}\right)^{\times n}$ inherits a product cell decomposition, with the boundary $n$-torus, $T^{n}=\left(S^{1}\right)^{\times n}$, as a subcomplex. For each subset $\sigma \subseteq[n]:=\{1, \ldots, n\}$, let $B_{\sigma}=\left\{z \in\left(D^{2}\right)^{\times n}|| z_{i} \mid=1\right.$ for $\left.i \notin \sigma\right\}$. Taking the union of the subcomplexes $B_{\sigma}$, indexed by the simplices of $K$, yields the moment-angle complex $\mathcal{Z}_{K}$. This cellular complex is always 2 -connected and has dimension $n+\ell+1$, where $\ell$ is the dimension of $K$.

It turns out that the algebraic topology of a moment-angle complex $\mathcal{Z}_{K}$, as embodied, for example, in the cohomology ring, the homotopy groups, and the Massey products, is intimately related to the combinatorics of the underlying simplicial complex $K$.

1.2. Cohomology ring and homotopy groups. Fix a coefficient field $\mathbb{k}$ of characteristic 0 . Let $S=\mathbb{k}\left[x_{1}, \ldots, x_{n}\right]$ be the polynomial ring with variables in degree 2 , and let $I$ be the ideal generated by all monomials corresponding to non-faces of $K$. As shown by Buchstaber and Panov [9], the cohomology ring of the moment-angle complex, $H^{*}\left(\mathcal{Z}_{K}, \mathbb{k}\right)$, is isomorphic to $\operatorname{Tor}^{S}(S / I, \mathbb{k})$, the Toralgebra of the Stanley-Reisner ring $S / I$.

Here, we compute the ranks of the homotopy groups of $\mathcal{Z}_{K}$ in terms of the homological algebra of $S / I$. A crucial ingredient is provided by the fibration $\mathcal{Z}_{K} \rightarrow \mathcal{D} \mathcal{J}(K) \rightarrow B T^{n}$, where $\mathcal{D} \mathcal{J}(K)$ is the Davis-Januszkiewicz space associated to $K$. The formality of this space - a result of Franz [22] for smooth toric fans, and Notbohm and Ray [43] in general-leads to the collapse of the Eilenberg-Moore spectral sequence for the path-fibration of $\mathcal{D} \mathcal{J}(K)$, thereby allowing us to compute $\pi_{*}\left(\mathcal{Z}_{K}\right) \otimes \mathbb{k}$ in terms of $\operatorname{Tor}^{S / I}(\mathbb{k}, \mathbb{k})$, the dual of the Yoneda algebra of $S / I$. 


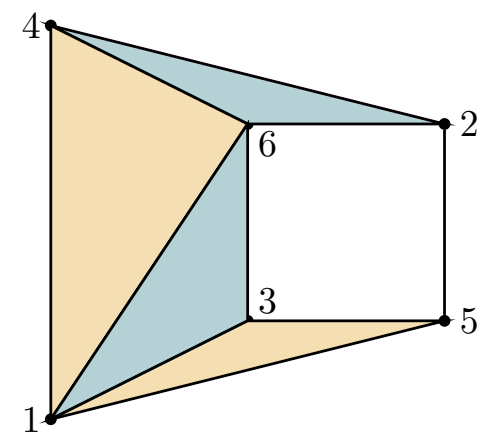

Figure 1. A simplicial complex $K$ for which $\mathcal{Z}_{K}$ is non-formal

The answer turns out to be particularly nice when $K$ is a flag complex, in which case $S / I$ is a Koszul algebra. Writing $h(H, s, t)$ for the bigraded Hilbert series of $H=\operatorname{Tor}^{S}(S / I, \mathbb{k})$, and making use of Koszul duality, we prove (Theorem 4.2.1):

$$
\prod_{r=1}^{\infty}\left(1-(-t)^{r}\right)^{(-1)^{r} \operatorname{rank} \pi_{r+1}\left(\mathcal{Z}_{K}\right)}=h(H, i \sqrt{t},-i \sqrt{t}) .
$$

This formula provides an effective method for computing the rational homotopy groups of such moment-angle complexes, at least in low degrees; see Examples 7.2.1 and 8.5.1.

1.3. Cellular cochains and Massey products. A key observation of Buchstaber and Panov [9] is that the cellular cochain complex $C_{\mathrm{cw}}^{*}\left(\mathcal{Z}_{K}, \mathbb{k}\right)$ comes endowed with a multiplication which makes it into a commutative differential (bi)graded algebra, quasi-isomorphic to the Koszul complex of $S / I$. An explicit formula for the cochain multiplication, in terms of pairings between full subcomplexes, can be found in [4].

Using this approach, Baskakov [5] constructs simplicial complexes $K$ for which the cellular cochain algebra $C_{\mathrm{cw}}^{*}\left(\mathcal{Z}_{K}, \mathbb{k}\right)$ has non-vanishing Massey triple products. In turn, this implies the non-formality of $\mathcal{Z}_{K}$; see $\S 5$ for more details.

We embark here on a more systematic study of Massey products in momentangle complexes. As a start, we characterize those simplicial complexes $K$ for which $\mathcal{Z}_{K}$ has a non-trivial Massey product in lowest possible degree, purely in combinatorial terms. In Theorem 6.1.1, we prove: there exist $\alpha, \beta, \gamma \in H^{3}\left(\mathcal{Z}_{K}, \mathbb{k}\right)$ such that $\langle\alpha, \beta, \gamma\rangle \neq 0$ precisely when the 1-skeleton of $K$ has an induced subgraph isomorphic to one of the five "obstruction" graphs listed in Figure 2. (The smallest such simplicial complex is depicted in Figure 1.) Moreover, all Massey products arising in this fashion are decomposable. 
On the other hand, we exhibit in $\S 8.5$ a simplicial complex $K$ for which $\mathcal{Z}_{K}$ carries an indecomposable triple Massey product; such a product manifests itself as a non-zero differential in the $E_{2}$ term of the Eilenberg-Moore spectral sequence for $\mathcal{Z}_{K}$.

1.4. Moment-angle manifolds. Now suppose $K$ is an $n$-vertex triangulation of the sphere $S^{\ell}$. Then, as shown by Buchstaber and Panov [9], the moment-angle complex $\mathcal{Z}_{K}$ is a smooth, compact, 2-connected manifold of dimension $n+\ell+1$. Some of these manifolds can be described in simple terms. For example, if the triangulation $K$ is dual to a polytope obtained from the hypercube by cutting corners, then $\mathcal{Z}_{K}$ is a connected sum of products of spheres.

In general, though, moment-angle manifolds can exhibit quite a complicated structure, both from the point of view of their cohomology ring and of their Massey products. We illustrate this point with an infinite family of triangulations of $S^{2}$ for which the corresponding manifolds have non-trivial triple Massey products. The simplest such manifold is constructed as follows: start with the square $\square$; form the deleted join of $\square$ with its Alexander dual, $\times$, to obtain $K=\operatorname{Bier}(\square)$, an 8-vertex Bier triangulation of $S^{2}$; the resulting moment-angle complex, $\mathcal{Z}_{K}$, is a non-formal, 11-dimensional manifold.

Asymptotically, almost all triangulations of $S^{2}$ yield non-formal moment-angle manifolds: see Theorem 8.3.1 for a precise statement. We detect this nonformality by means of decomposable Massey products. Using the Bier sphere construction, we exhibit in $\S 8.6$ a 16 -vertex triangulation $K$ of $S^{6}$ for which $\mathcal{Z}_{K}$ has an indecomposable Massey product.

1.5. Compact, complex, non-Kähler manifolds. Classical constructions of complex manifolds, due to Hopf and Calabi-Eckmann were generalized in recent years by López de Medrano-Verjovsky [39] and Meersseman [38]. These authors define a large class of compact complex manifolds admitting no Kähler structure. A complex manifold $N$ arises via the LVM construction as the leaf space of a foliation of $\mathbb{C} \mathbb{P}^{n-1}$, given by a suitable linear action of $\mathbb{C}^{m}$ on $\mathbb{C}^{n}$, with $n>2 m$. If $n=2 m+1$, then $N$ is a complex torus; otherwise, $N$ is non-symplectic, and thus non-Kähler.

It turns out that the LVM construction is very much related to that of the moment-angle complexes. We find some parallels and applications both ways, especially in connection with recent work of Bosio and Meersseman [8]. In particular, we explain the absence of Kähler structure for certain LVM manifolds via the presence of non-vanishing Massey products in the corresponding momentangle complex. 
1.6. Subspace arrangements. Much is known about the relationship between the combinatorics of a complex subspace arrangement (as encoded in its ranked intersection lattice) and the topology of its complement. In [25], Goresky and MacPherson gave a formula for the Betti numbers of the complement; the cupproducts in cohomology were computed by Deligne-Goresky-MacPherson [14] and de Longueville-Schultz [33].

Determining the homotopy type of the complement remains a challenging problem. If the intersection lattice is geometric, then, as shown by Feichtner and Yuzvinsky [19], the complement is formal; thus, its rational homotopy type is determined by the (ranked) intersection lattice. Using our approach, we see that the complement of a subspace arrangement is not formal in general: non-vanishing triple Massey products are not detected by the cohomology ring. The simplest such example is the arrangement of coordinate subspaces in $\mathbb{C}^{6}$ determined by the above simplicial complex: $\mathcal{A}=\left\{H_{1}, \ldots, H_{5}\right\}$, where $H_{i}=\left\{z \in \mathbb{C}^{6} \mid z_{i}=z_{i+1}=0\right\}$.

\section{The MOMENT-ANGLE FUnCTOR}

2.1. Generalized moment-angle complexes. We start with a generalization of the notion of moment-angle complex, due to Strickland [51], cf. [9, 4, 44].

Definition 2.1.1. Let $X$ be a space, and $A \subset X$ a non-empty subspace. Given a simplicial complex $K$ on vertex set $[n]$, define $\mathcal{Z}_{K}(X, A)$ to be the following subspace of the cartesian product $X^{\times n}$ :

$$
\mathcal{Z}_{K}(X, A)=\bigcup_{\sigma \in K}(X, A)^{\sigma},
$$

where $(X, A)^{\sigma}=\left\{x \in X^{\times n} \mid x_{i} \in A\right.$ if $\left.i \notin \sigma\right\}$.

In other words, $\mathcal{Z}_{K}(X, A)$ is the colimit of the diagram of spaces $\left\{(X, A)^{\sigma}\right\}$, indexed by the category whose objects are the simplices of $K$ (including the empty simplex), and whose morphisms are the inclusions between those simplices; see $[46,43]$.

Note that if $A=X$, or $K=\Delta^{n-1}$ is a simplex, then $\mathcal{Z}_{K}(X, A)=X^{\times n}$; at the other extreme, $\mathcal{Z}_{\emptyset}(X, A)=A^{\times n}$. From the definition, if $L$ is a subcomplex of $K$ on the same vertex set, then $\mathcal{Z}_{L}(X, A)$ is a subspace of $\mathcal{Z}_{K}(X, A)$; in particular, $A^{\times n} \subset \mathcal{Z}_{K}(X, A) \subset X^{\times n}$.

If $X$ is a finite-type CW-complex, and $A$ is a subcomplex, then $\mathcal{Z}_{K}(X, A)$ is a subcomplex of the product complex $X^{\times n}$; if $X$ is a finite CW-complex, $\mathcal{Z}_{K}(X, A)$ is also a finite $\mathrm{CW}$-complex.

Example 2.1.2. If $(X, *)$ is a pointed space, we will simply write $\mathcal{Z}_{K}(X):=$ $\mathcal{Z}_{K}(X, *)$. Particularly noteworthy are the following examples. 
(1) If $K$ is a discrete set of $n$ points, then $\mathcal{Z}_{K}(X)=\bigvee^{n} X$, the wedge of $n$ copies of $X$.

(2) If $K=\partial \Delta^{n-1}$ is the boundary of a simplex, then $\mathcal{Z}_{K}(X)$ is the fat wedge of $n$ copies of $X$.

(3) If $\Delta(\Gamma)$ is the flag complex of a simple graph $\Gamma$, then $\mathcal{Z}_{\Delta(\Gamma)}\left(S^{1}\right)=$ $K\left(G_{\Gamma}, 1\right)$, an Eilenberg-MacLane space for the right-angled Artin group $G_{\Gamma} ;$ see $[10,40]$.

(4) If $X$ is the classifying space $B S^{1}=\mathbb{C P}^{\infty}$, then $\mathcal{Z}_{K}\left(B S^{1}\right)=\mathcal{D} \mathcal{J}(K)$, the Davis-Januszkiewicz space associated to $K$; see $[12,9]$, and also $\S 2.3$.

Example 2.1.3. In the case when $(X, A)=\left(D^{2}, S^{1}\right)$, we obtain the usual moment-angle complex, $\mathcal{Z}_{K}=\mathcal{Z}_{K}\left(D^{2}, S^{1}\right)$, which is our main object of study. Note that $\operatorname{dim} \mathcal{Z}_{K}=\operatorname{dim} K+n+1$. Here are some samples, to get started.

(1) If $K=\partial \Delta^{n-1}$, then $\mathcal{Z}_{K}=S^{2 n-1}$.

(2) If $K$ a discrete set of $n$ points, then $\mathcal{Z}_{K} \simeq \bigvee_{k=2}^{n}(k-1)\left(\begin{array}{l}n \\ k\end{array}\right) S^{k+1}$; see [27].

The moment-angle construction behaves nicely with respect to joins of simplicial complexes.

Lemma 2.1.4. For any simplicial complexes $K, K^{\prime}$ and pair of spaces $(X, A)$, there is a homeomorphism

$$
\mathcal{Z}_{K * K^{\prime}}(X, A) \stackrel{\cong}{\longrightarrow} \mathcal{Z}_{K}(X, A) \times \mathcal{Z}_{K^{\prime}}(X, A) .
$$

Proof. View the respective moment-angle complexes as subspaces $\mathcal{Z}_{K}(X, A) \subset$ $X^{\times n}, \mathcal{Z}_{K^{\prime}}(X, A) \subset X^{\times n^{\prime}}$, and $\mathcal{Z}_{K * K^{\prime}}(X, A) \subset X^{\times\left(n+n^{\prime}\right)}$. The natural identi-

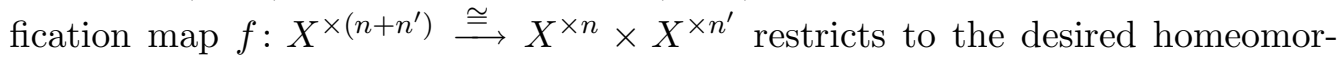
phism.

Remark 2.1.5. One may slightly generalize Definition 2.1.1, and allow the vertex set $V$ of $K$ to be a (possibly proper) subset of $[n]$. The resulting subspace of $X^{\times n}$, call it $\mathcal{Z}_{K, n}(X, A)$, is then homeomorphic to $\mathcal{Z}_{K}(X, A) \times A^{\times(n-|V|)}$. For example, $\mathcal{Z}_{\emptyset, n}(X, A)=A^{\times n}$, and $\mathcal{Z}_{K, n}\left(D^{2}, S^{1}\right)=\mathcal{Z}_{K}\left(D^{2}, S^{1}\right) \times T^{n-|V|}$, where we recall $T^{m}$ is the $m$-torus. We will not pursue this generality, except briefly in $\S 8.4$.

2.2. Naturality. The $\mathcal{Z}$ construction enjoys functoriality properties in both arguments, which we now summarize.

Lemma 2.2.1. Let $f:(X, A) \rightarrow(Y, B)$ be a map of pairs. Then $f^{\times n}: X^{\times n} \rightarrow$ $Y^{\times n}$ restricts to a map

$$
\mathcal{Z}_{K}(f): \mathcal{Z}_{K}(X, A) \rightarrow \mathcal{Z}_{K}(Y, B) .
$$

Moreover, $\mathcal{Z}_{K}(g \circ f)=\mathcal{Z}_{K}(g) \circ \mathcal{Z}_{K}(f)$. And, if $f$ is a cellular map between $C W$ pairs, then $\mathcal{Z}_{K}(f)$ is also a cellular map. 
Now suppose $F:(X, A) \times I \rightarrow(Y, B)$ is a relative homotopy. Then the product homotopy $\left(X^{\times n}, A^{\times n}\right) \times I \rightarrow\left(Y^{\times n}, B^{\times n}\right)$ restricts to a homotopy $\mathcal{Z}_{K}(X, A) \times I \rightarrow$ $\mathcal{Z}_{K}(Y, B)$. Thus, $\mathcal{Z}_{K}$ is a homotopy functor: the homotopy type of $\mathcal{Z}_{K}(X, A)$ depends only on the relative homotopy type of the pair $(X, A)$. Also note the following: if $(X, A)$ deform-retracts onto $(Y, B)$, then $\mathcal{Z}_{K}(X, A)$ deform-retracts onto $\mathcal{Z}_{K}(Y, B)$.

The following observation is due to Strickland [51].

Lemma 2.2.2. Let $X$ be a commutative topological monoid, and $A$ a sub-monoid. Suppose $f: K \rightarrow L$ is simplicial map between simplicial complexes on vertex sets $[n]$ and $[m]$. Then, the map $\tilde{f}: X^{\times n} \rightarrow X^{\times m}$ defined by $\tilde{f}(x)_{j}=\prod_{i: f(i)=j} x_{i}$ restricts to a map

$$
\mathcal{Z}_{f}(X, A): \mathcal{Z}_{K}(X, A) \rightarrow \mathcal{Z}_{L}(X, A)
$$

Clearly, $\mathcal{Z}_{g \circ f}(X, A)=\mathcal{Z}_{g}(X, A) \circ \mathcal{Z}_{f}(X, A)$. Moreover, if $(X, A)$ is a CW pair, then $\mathcal{Z}_{f}(X, A)$ is a cellular map.

The Lemma applies to the pair $\left(D^{2}, S^{1}\right)$, with monoid structure defined by complex multiplication. This yields a moment-angle functor from finite simplicial complexes $K$ and simplicial maps $f: K \rightarrow L$ to finite $\mathrm{CW}$-complexes $\mathcal{Z}_{K}$ and cellular maps $\mathcal{Z}_{f}: \mathcal{Z}_{K} \rightarrow \mathcal{Z}_{L}$.

There is a second functorial property that holds for all pairs $(X, A)$, but only certain simplicial maps. Recall that if $K$ is a subcomplex of $L$, then $K$ is a full subcomplex if every simplex of $L$ on vertices of $K$ is also a simplex of $K$.

Lemma 2.2.3. Let $(X, A)$ be a pair of spaces, and $* \in A$ a basepoint. Suppose $f: K \hookrightarrow L$ is the inclusion of a full subcomplex, sending vertex set $[n]$ to $[m]$. Then:

(i) The canonical projection $X^{\times m} \rightarrow X^{\times n}$ restricts to a surjective map $\mathcal{Z}^{f}(X, A): \mathcal{Z}_{L}(X, A) \rightarrow \mathcal{Z}_{K}(X, A)$.

(ii) The map $\hat{f}: X^{\times n} \hookrightarrow X^{\times m}$, defined by $\hat{f}(x)_{j}=x_{i}$ if $f(i)=j$, and $\hat{f}(x)_{j}=$ $*$, otherwise, restricts to an injective map $\mathcal{Z}_{f, *}(X, A): \mathcal{Z}_{K}(X, A) \hookrightarrow$ $\mathcal{Z}_{L}(X, A)$.

Moreover, $\mathcal{Z}^{f}(X, A) \circ \mathcal{Z}_{f, *}(X, A)=\mathrm{id}$.

In the case when $X$ is a commutative monoid, $A$ is a sub-monoid, and $* \in A$ is the unit, we have $\mathcal{Z}_{f, *}(X, A)=\mathcal{Z}_{f}(X, A)$.

2.3. The homotopy fibre interpretation. An important property of the functor $\mathcal{Z}_{K}$ is that it takes certain relative fibrations to fibrations. We make this observation more precise in the following Lemma. As an application, we obtain two useful fibrations (Lemmas 2.3.2 and 2.3.3) generalizing those of Buchstaber and Panov. 
Lemma 2.3.1. Let $p:\left(E, E^{\prime}\right) \rightarrow\left(B, B^{\prime}\right)$ be a map of pairs, such that both $p: E \rightarrow$ $B$ and $\left.p\right|_{E^{\prime}}: E^{\prime} \rightarrow B^{\prime}$ are fibrations, with fibres $F$ and $F^{\prime}$, respectively. Suppose that either $F=F^{\prime}$ or $B=B^{\prime}$. Then the product fibration, $p^{\times n}: E^{\times n} \rightarrow B^{\times n}$, restricts to a fibration

$$
\mathcal{Z}_{K}\left(F, F^{\prime}\right) \longrightarrow \mathcal{Z}_{K}\left(E, E^{\prime}\right) \stackrel{\mathcal{Z}_{K}(p)}{\longrightarrow} \mathcal{Z}_{K}\left(B, B^{\prime}\right)
$$

Moreover, if $\left(F, F^{\prime}\right) \rightarrow\left(E, E^{\prime}\right) \rightarrow\left(B, B^{\prime}\right)$ is a relative bundle (with structure group $G$ ), and either $F=F^{\prime}$ or $B=B^{\prime}$, then (3) is also a bundle (with structure group $\left.G^{\times n}\right)$.

Proof. Suppose first that $B=B^{\prime}$; then $\mathcal{Z}_{K}\left(B, B^{\prime}\right)=B^{\times n}$. The fibre of $\left.p^{\times n}\right|_{\mathcal{Z}_{K}\left(E, E^{\prime}\right)}$ equals

$$
\begin{aligned}
F^{\times n} \cap \mathcal{Z}_{K}\left(E, E^{\prime}\right) & =\bigcup_{\sigma \in K} F^{\times n} \cap\left(E, E^{\prime}\right)^{\sigma} \\
& =\bigcup_{\sigma \in K}\left(F, F^{\prime}\right)^{\sigma} \quad \text { since } F \cap E^{\prime}=F^{\prime} \\
& =\mathcal{Z}_{K}\left(F, F^{\prime}\right) .
\end{aligned}
$$

The homotopy lifting property (or the bundle map property) for $\mathcal{Z}_{K}(p)$ follows from those of $p$ and $\left.p\right|_{E^{\prime}}$.

Similarly, if $F=F^{\prime}$, it is straightforward to check that $\mathcal{Z}_{K}\left(E, E^{\prime}\right)$ is the pullback of $E^{\times n}$ along the inclusion of $\mathcal{Z}_{K}\left(B, B^{\prime}\right)$ into $B^{\times n}$.

For a topological group $G$, denote by $G \rightarrow E G \rightarrow B G$ the universal principal $G$-bundle, with $E G$ a contractible space endowed with a free $G$-action, and $B G$ the orbit space. We may view $G$ as a subspace of $E G$ (the orbit of a basepoint *). If $X$ is a $G$-space, denote by $E G \times_{G} X$ the Borel construction, and by $X \rightarrow E G \times_{G} X \rightarrow B G$ the associated bundle.

Now suppose $A$ is a $G$-invariant subspace of $X$. The $G$-action on the pair $(X, A)$ extends canonically to an action of the product group $G^{\times n}$ on the pair $\left(X^{\times n}, A^{\times n}\right)$. It is readily checked that this $G^{\times n}$-action preserves the subspace $\mathcal{Z}_{K}(X, A) \subset X^{\times n}$.

Lemma 2.3.2. With notation as above, we have:

(1) $E G^{\times n} \times_{G^{\times n}} \mathcal{Z}_{K}(E G, G) \simeq \mathcal{Z}_{K}(B G)$.

(2) The homotopy fibre of the inclusion $\mathcal{Z}_{K}(B G) \hookrightarrow B G^{\times n}$ is $\mathcal{Z}_{K}(E G, G)$. In other words, we have a fibration sequence $\mathcal{Z}_{K}(E G, G) \rightarrow \mathcal{Z}_{K}(B G) \rightarrow$ $B G^{\times n}$.

Proof. The free action of $G$ on $E G$ gives an associated bundle $E G \rightarrow E G \times_{G}$ $E G \rightarrow B G$. Restricting each fibre $E G$ to the subspace $G$, we obtain a sub-bundle 
$G \rightarrow E G \times * \rightarrow B G$, isomorphic to the classifying bundle. From Lemma 2.3.1, since the base spaces agree, we obtain the fibre bundle sequence in the top row of the following diagram:

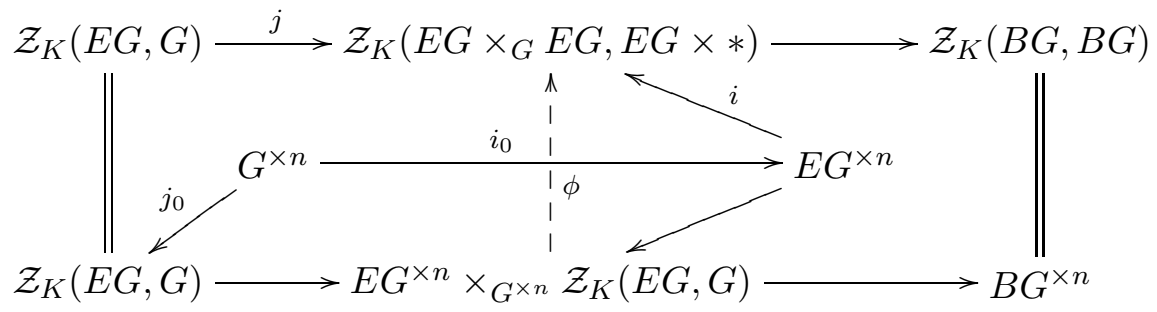

The bottom row is the associated bundle to $G^{\times n} \rightarrow E G^{\times n} \rightarrow B G^{\times n}$ and the natural action of $G^{\times n}$ on $\mathcal{Z}_{K}(E G, G) \subset E G^{\times n}$. The total space of this bundle is the pushout of $E G^{\times n}$ and $\mathcal{Z}_{K}(E G, G)$ along the natural inclusions $i_{0}$ and $j_{0}$ of $G^{\times n}$ into each.

Identifying $E G^{\times n}$ with $(E G \times *)^{\times n}$ yields an inclusion $i$ into $\mathcal{Z}_{K}\left(E G \times_{G}\right.$ $E G, E G \times *)$. We also have the inclusion $j$ of $\mathcal{Z}_{K}(E G, G)$ into that space, as the typical fiber of the top bundle. It is readily seen that $i$ and $j$ agree on the common subspace $G^{\times n}$; therefore, there is a map $\phi$ as indicated in the diagram. Since both $i$ and $j$ are $G^{\times n}$-equivariant, $\phi$ is a bundle map. Since the restriction of $\phi$ to each fibre is the identity, we conclude that $\phi$ is a homeomorphism.

Now note that $\left(E G \times_{G} E G, E G \times *\right) \simeq(B G, *)$, and so $\mathcal{Z}_{K}\left(E G \times_{G} E G, E G \times\right.$ $*) \simeq \mathcal{Z}_{K}(B G)$, by the remark following Lemma 2.2.1. Putting things together finishes the proof of claim (1). Claim (2) follows at once.

Lemma 2.3.3. There is a fibre bundle sequence

$$
G^{\times n} \rightarrow \mathcal{Z}_{K}(E G, G) \rightarrow \mathcal{Z}_{K}(B G) .
$$

Proof. Take the universal bundle $G \rightarrow E G \rightarrow B G$ and restrict the base to the basepoint $*$ to obtain a relative $G$-bundle $(G, G) \rightarrow(E G, G) \rightarrow(B G, *)$. Then apply Lemma 2.3.1, this time where the two fibres agree.

For the circle $S^{1}$, the universal bundle is the Hopf fibration $S^{1} \rightarrow S^{\infty} \rightarrow \mathbb{C P}^{\infty}$. Thus, a classifying space for the torus $T^{n}=\left(S^{1}\right)^{\times n}$ is $B T^{n}=\left(\mathbb{C P}^{\infty}\right)^{\times n}$. The circle acts on the pair $\left(D^{2}, S^{1}\right)$ by rotation. As noted above, this $S^{1}$-action extends to a $T^{n}$-action on the moment-angle complex $\mathcal{Z}_{K}=\mathcal{Z}_{K}\left(D^{2}, S^{1}\right)$. Finally, observe that $\left(E S^{1}, S^{1}\right) \simeq\left(D^{2}, S^{1}\right)$; thus, $\mathcal{Z}_{K}\left(E S^{1}, S^{1}\right) \simeq \mathcal{Z}_{K}$.

The Davis-Januszkiewicz space associated to the simplicial complex $K$ is, by definition, the Borel construction on the moment-angle complex $\mathcal{Z}_{K}$, viewed as a $T^{n}$-space:

$$
\mathcal{D} \mathcal{J}(K)=E T^{n} \times_{T^{n}} \mathcal{Z}_{K}
$$


We thus have a fibre bundle sequence $\mathcal{Z}_{K} \rightarrow \mathcal{D} \mathcal{J}(K) \rightarrow B T^{n}$. It follows that the homotopy fibre of the inclusion $\mathcal{Z}_{K} \hookrightarrow \mathcal{D} \mathcal{J}(K)$ is $T^{n}$, a particular case of Lemma 2.3.3.

From Lemma 2.3.2, we recover the following result of Buchstaber and Panov [9].

Corollary 2.3.4. The following hold:

(1) $\mathcal{D} \mathcal{J}(K) \simeq \mathcal{Z}_{K}\left(B S^{1}\right)$

(2) The homotopy fibre of the inclusion $\mathcal{Z}_{K}\left(B S^{1}\right) \hookrightarrow\left(B S^{1}\right)^{\times n}$ is $\mathcal{Z}_{K}$.

(3) We have a fibration sequence $\mathcal{Z}_{K} \rightarrow \mathcal{Z}_{K}\left(B S^{1}\right) \rightarrow B T^{n}$.

\section{Cohomology and the Stanley-Reisner Ring}

In this section, we outline Buchstaber and Panov's computation of the cohomology ring of a moment-angle complex $\mathcal{Z}_{K}$. We work over a fixed coefficient ring which is, by default, the integers $\mathbb{Z}$. We will use $\mathbb{k}$ to denote an arbitrary field of characteristic zero.

3.1. Cohomology ring of $\mathcal{Z}_{K}(X)$. Consider a pointed space $(X, *)$. Each inclusion of simplices $\sigma \subseteq \tau$ in $K$ gives rise to an inclusion $(X, *)^{\sigma} \hookrightarrow(X, *)^{\tau}$. Such an inclusion has a left inverse, obtained by mapping $X$ to $*$ in coordinates from $\tau \backslash \sigma$, and by mapping $X$ to $X$ identically elsewhere. It follows that the induced maps in cohomology, $H^{*}\left((X, *)^{\tau}, \mathbb{Z}\right) \rightarrow H^{*}\left((X, *)^{\sigma}, \mathbb{Z}\right)$, are split surjections. Due to this splitting, the Mayer-Vietoris spectral sequence degenerates at its $E_{2}$ term, yielding an isomorphism

$$
H^{*}\left(\mathcal{Z}_{K}(X), \mathbb{Z}\right) \cong \lim _{\sigma \in K} H^{*}\left((X, *)^{\sigma}, \mathbb{Z}\right)
$$

Lemma 3.1.1. For any pair $(X, *)$, the inclusion $j: \mathcal{Z}_{K}(X, *) \hookrightarrow \mathcal{Z}_{K}(X, X)=$ $X^{\times n}$ induces a surjection of rings

$$
j^{*}: H^{*}\left(X^{\times n}, \mathbb{Z}\right) \rightarrow H^{*}\left(\mathcal{Z}_{K}(X), \mathbb{Z}\right)
$$

Proof. We use the same splitting argument. For each $\sigma \in K$, the inclusion $(X, *)^{\sigma} \hookrightarrow(X, X)^{\sigma}$ has a left inverse, so the inclusion $j: \mathcal{Z}_{K}(X, *) \hookrightarrow \mathcal{Z}_{K}(X, X)$ does too. Therefore, $j$ induces a split surjection in cohomology.

If $X$ is a CW-complex, the inclusion $j$ above is easily seen to be cellular. Its image consists of cells $e$ for which there exists a simplex $\sigma \in K$ with $e_{i}=*$ for all $i \notin \sigma$. If further, $X$ has a minimal cell structure -i.e., if the number of $p$-cells of $X$ equals $b_{p}(X)$, for all $p$-more can be said: the kernel of $j^{*}$ is spanned (additively) by the dual basis to the complement of the image of $j$; that is, those $e^{*}$ for which, for some non-face $\sigma,\left(e^{*}\right)_{i} \neq 1$ for all $i \in \sigma$. 
Two important cases are $X=S^{1}$ and $X=B S^{1}$; see Section 2.1. In either case, associate with a simplex $\sigma=\left\{i_{1}, \ldots, i_{k}\right\}$ a square-free monomial $x_{\sigma}=$ $x_{i_{1}} x_{i_{2}} \cdots x_{i_{k}}$.

Theorem 3.1.2 ([12, 9, 30]). If $K$ is a simplicial complex on $n$ vertices,

(1) $H^{*}\left(\mathcal{Z}_{K}\left(S^{1}\right), \mathbb{Z}\right) \cong \bigwedge\left[x_{1}, \ldots, x_{n}\right] / J_{K}$, and

(2) $H^{*}\left(\mathcal{Z}_{K}\left(B S^{1}\right), \mathbb{Z}\right) \cong \mathbb{Z}\left[x_{1}, \ldots, x_{n}\right] / I_{K}$,

where $J_{K}$ and $I_{K}$ are the ideals in the exterior and polynomial algebras, respectively, generated by all monomials $x_{\sigma}$ for which $\sigma$ is not a face of $K$.

Proof. Note that $\mathbb{C} P^{\infty}=B S^{1}$ and $S^{1}$ both have a minimal cell structure. We have $H^{*}\left(\left(B S^{1}\right)^{\times n}, \mathbb{Z}\right) \cong \mathbb{Z}\left[x_{1}, \ldots, x_{n}\right]$, where the generator $x_{i}$ is dual to the 2-cell in the $i$ th coordinate, and $H^{*}\left(\left(S^{1}\right)^{\times n}, \mathbb{Z}\right)$ is an exterior algebra with degree-1 generators.

By Lemma 3.1.1, then, it is enough to notice that the dual basis to the cells in $\mathcal{Z}_{K}\left(B S^{1}\right)$ that are not in $\left(B S^{1}\right)^{\times n}$ consists exactly of the monomials in $I_{K}$, and similarly for $S^{1}$.

Let $E$ and $S$ denote the exterior and polynomial rings above, with generators in degree 1 and 2, respectively. That is, the cohomology of the Davis-Januszkiewicz space $\mathcal{D} \mathcal{J}(K)=\mathcal{Z}_{K}\left(B S^{1}\right)$ is simply the Stanley-Reisner ring of $K$ (see $\left.[12,9,44]\right)$ :

$$
H^{*}(\mathcal{D} \mathcal{J}(K), \mathbb{Z})=S / I_{K}
$$

When $K=\Delta(\Gamma)$ is the flag complex of a graph $\Gamma$, recall from Section 2.1 that $\mathcal{Z}_{K}\left(S^{1}\right)$ is a $K\left(G_{\Gamma}, 1\right)$ space for the right-angled Artin group $G_{\Gamma}$. Thus, the cohomology of $K\left(G_{\Gamma}, 1\right)$ is simply the exterior Stanley-Reisner ring of $K$ (see $[30])$ :

$$
H^{*}\left(G_{\Gamma}, \mathbb{Z}\right)=E / J_{K}
$$

3.2. Cohomology ring of $\mathcal{Z}_{K}$. Recall from Lemma 2.3.2 that, for any compact Lie group $G$, there is a fibration sequence $\mathcal{Z}_{K}(E G, G) \rightarrow \mathcal{Z}_{K}(B G) \rightarrow \mathcal{Z}_{K}\left(G^{\times n}\right)$. With the additional assumption that $G$ is connected, we have an Eilenberg-Moore spectral sequence

$$
\begin{aligned}
E_{2}^{p, q} & =\operatorname{Tor}_{p}^{H^{*}\left(B G^{\times n}, \mathbb{Z}\right)}\left(H^{*}\left(\mathcal{Z}_{K}(B G), \mathbb{Z}\right), H^{*}(*, \mathbb{Z})\right)^{q} \\
& =\operatorname{Tor}_{p}^{H^{*}\left(B G^{\times n}, \mathbb{Z}\right)}\left(H^{*}\left(\mathcal{Z}_{K}(B G), \mathbb{Z}\right), \mathbb{Z}\right)^{q} \Rightarrow H^{p+q}\left(\mathcal{Z}_{K}(E G, G), \mathbb{Z}\right),
\end{aligned}
$$

in which resolutions are graded by total degree $q$.

In the case of $G=S^{1}$, the cohomology ring $H^{*}\left(B G^{\times n}, \mathbb{Z}\right)$ is the polynomial ring $S$ with generators in degree 2 . We have a homotopy equivalence $\mathcal{Z}_{K}\left(E S^{1}, S^{1}\right) \simeq \mathcal{Z}_{K}\left(D^{2}, S^{1}\right)$, by the discussion in Section 2.1. As stated in [9], 
the Eilenberg-Moore spectral sequence (9) degenerates at $E_{2}$; moreover, one has an isomorphism of rings between the cohomology of the moment-angle complex, $\mathcal{Z}_{K}=\mathcal{Z}_{K}\left(D^{2}, S^{1}\right)$, and the Tor algebra of $S / I_{K}$ :

$$
H^{*}\left(\mathcal{Z}_{K}, \mathbb{Z}\right)=\operatorname{Tor}^{S}\left(S / I_{K}, \mathbb{Z}\right) .
$$

The argument from [9], completed in [44], relies on the cellular algebra constructed in [6]: see the discussion in $\S 5.3$, in particular equation (21).

Returning now to the more general situation, let $G$ be an arbitrary compact, connected Lie group, and fix a field $\mathbb{k}$ of characteristic 0. By a classical result of Borel, $H^{*}(B G, \mathbb{k})=H^{*}(B T, \mathbb{k})^{W}$, where $T$ is a maximal torus, and $W$ is the Weyl group; consequently, $H^{*}(B G, \mathbb{k})$ is a polynomial algebra. It follows that $H^{*}(B G, \mathbb{k})$, with 0 as the differential, is a minimal model for $B G$, and so $B G$ (and $B G^{\times n}$ ) are formal spaces; see [52] and the discussion in $\S 5.2$.

Question 3.2.1. In this respect, the following interrelated questions arise naturally:

(1) For which Lie groups $G$ does the Eilenberg-Moore spectral sequence (9) degenerate at $E_{2}$ ?

(2) When (1) holds, is the additive isomorphism $E_{2} \cong H^{*}\left(\mathcal{Z}_{K}(E G, G), \mathbb{k}\right)$ an isomorphism of rings?

(3) For which groups $G$ is the space $\mathcal{Z}_{K}(B G)$ formal? (This is established for $G=S^{1}$ in [43].)

3.3. Functoriality in $K$. Let $(X, A)$ be a pair of spaces, $L$ a simplicial complex, and $K \subset L$ a full subcomplex. By Lemma 2.2.3, the moment-angle complex $\mathcal{Z}_{K}(X, A)$ is a subcomplex of $\mathcal{Z}_{L}(X, A)$, with the inclusion map admitting a retraction $\mathcal{Z}_{L}(X, A) \rightarrow \mathcal{Z}_{K}(X, A)$. As a consequence, we obtain the following.

Proposition 3.3.1. If $K$ is a full subcomplex of $L$, then $H^{*}\left(\mathcal{Z}_{K}(X, A), \mathbb{Z}\right)$ splits multiplicatively as direct summand of $H^{*}\left(\mathcal{Z}_{L}(X, A), \mathbb{Z}\right)$.

One can say more for pairs $(X, *)$ for which $X$ is a topological monoid, with $*$ as the unit. For that, we need to switch again to coefficients in our field $\mathbb{k}$. By the Künneth formula, $H^{*}(X \times X, \mathbb{k}) \cong H^{*}(X, \mathbb{k}) \otimes H^{*}(X, \mathbb{k})$. It follows that $H^{*}(X, \mathbb{k})$ is a Hopf algebra, where the multiplication $\mu: X \times X \rightarrow X$ induces a coproduct $H^{*}(X, \mathbb{k}) \rightarrow H^{*}(X, \mathbb{k}) \otimes H^{*}(X, \mathbb{k})$ given by $x \mapsto x \otimes 1+1 \otimes x$ for indecomposable $x$; see [42].

Now suppose that $K$ and $L$ are simplicial complexes on $[n]$ and $[m]$, respectively, and $\rho: K \rightarrow L$ is a map of simplicial complexes. Then $\mathcal{Z}_{\rho}: \mathcal{Z}_{K}(X, X) \rightarrow$ $\mathcal{Z}_{L}(X, X)$ induces a ring homomorphism $\rho^{*}=H^{*}\left(\mathcal{Z}_{\rho}\right): H^{*}(X, \mathbb{k})^{\otimes m} \rightarrow H^{*}(X, \mathbb{k})^{\otimes n}$ given by

$$
\rho^{*}\left(x^{(j)}\right)=\sum_{i: \rho(i)=j} x^{(i)}
$$


where $x^{(i)}$ denotes the element $1 \otimes \cdots \otimes 1 \otimes x \otimes 1 \cdots \otimes 1$, with $x$ in the $i$ th position.

Lemma 3.3.2. If $\rho: K \rightarrow L$ is a map of simplicial complexes as above, and $X=$ $S^{1}$ or $X=B S^{1}$, then the homomorphism $\rho^{*}: H^{*}\left(\mathcal{Z}_{K}(X), \mathbb{k}\right) \rightarrow H^{*}\left(\mathcal{Z}_{L}(X), \mathbb{k}\right)$ is given by $\rho^{*}\left(x_{j}\right)=\sum_{i: \rho(i)=j} x_{i}$.

Proof. From Lemma 3.1.1, this homomorphism is induced from

$$
\rho^{*}: H^{*}\left(\mathcal{Z}_{K}(X, X), \mathbb{k}\right) \rightarrow H^{*}\left(\mathcal{Z}_{L}(X, X), \mathbb{k}\right),
$$

which is given by (11). The claim follows.

For $X=B S^{1}$, this is a result of Panov [44].

\section{Homotopy groups And Koszul algebras}

In this section, we compute the ranks of the homotopy groups of a momentangle complex $\mathcal{Z}_{K}$ in terms of the homological algebra of the Stanley-Reisner ring $S / I_{K}$. The answer turns out to be particularly nice in the case when $K$ is a flag complex (equivalently, $S / I_{K}$ is a Koszul algebra). For related results, see the work in progress by Panov and Ray [45].

Throughout this section, $\mathbb{k}$ continues to denote a field of characteristic zero.

4.1. The homotopy Lie algebra. For a simply-connected, finite-type CWcomplex $X$, denote by $\Omega X$ the space of Moore loops at the basepoint $*$, endowed with the compact-open topology. The homotopy Lie algebra of $X$ (over $\mathbb{k}$ ) is the graded $\mathbb{k}$-vector space

$$
\mathfrak{g}_{X}=\bigoplus_{r \geq 1} \pi_{r}(\Omega X) \otimes \mathbb{k}
$$

endowed with the graded Lie algebra structure coming from the Whitehead product on $\pi_{*}(X)$ via the boundary map in the path fibration $\Omega X \rightarrow P X \rightarrow X$. By the Milnor-Moore theorem [42], the universal enveloping algebra of $\mathfrak{g}_{X}$ is isomorphic, as a Hopf algebra, to $H_{*}(\Omega X, \mathbb{k})$.

Now let $K$ be a simplicial complex on vertex set $[n]$ with no isolated vertices. From its construction as a subcomplex of $B T^{n}$, it is readily seen that $\mathcal{Z}_{K}\left(B T^{n}\right)$ shares the same 3 -skeleton with $B T^{n}=K\left(\mathbb{Z}^{n}, 2\right)$; hence, $\pi_{1}(\mathcal{D} \mathcal{J}(K))=0$ and $\pi_{2}(\mathcal{D} \mathcal{J}(K))=\mathbb{Z}^{n}$. From the long exact homotopy sequence for the fibration

$$
\mathcal{Z}_{K} \rightarrow \mathcal{D} \mathcal{J}(K) \rightarrow B T^{n}
$$

it follows that $\mathcal{Z}_{K}$ is 2-connected, and $\pi_{q}\left(\mathcal{Z}_{K}\right) \cong \pi_{q}(\mathcal{D} \mathcal{J}(K))$ for all $q \geq 3$, a result of Buchstaber and Panov [9]. In fact, as these authors note, if $K$ is $k$-neighborly (i.e., every $k$-tuple in $[n]$ is a simplex in $K$ ), then $\mathcal{Z}_{K}$ is $2 k$-connected. 
For convenience, denote the homotopy Lie algebras of $\mathcal{D} \mathcal{J}(K)$ and $\mathcal{Z}_{K}$ by $\mathfrak{g}_{\mathcal{D} \mathcal{J}}$ and $\mathfrak{g}_{\mathcal{Z}}$, respectively. Using the fibration (13), we obtain a short exact sequence of graded Lie algebras,

$$
0 \rightarrow \mathfrak{g}_{\mathcal{Z}} \rightarrow \mathfrak{g}_{\mathcal{D} \mathcal{J}} \rightarrow L_{n} \rightarrow 0
$$

where $L_{n}$ denotes the abelian Lie algebra of rank $n$ generated in degree 1. Taking Hilbert series of enveloping Lie algebras, we obtain

$$
h\left(U\left(\mathfrak{g}_{\mathcal{D} \mathcal{J}}\right), t\right)=h\left(U\left(\mathfrak{g}_{\mathcal{Z}}\right), t\right)(1+t)^{n} .
$$

Proposition 4.1.1. Let $K$ be a simplicial complex with $n$ vertices and $S / I$ the corresponding Stanley-Reisner ring. Then the ranks $\phi_{r}=\operatorname{rank} \pi_{r}\left(\mathcal{Z}_{K}\right)$ of the homotopy groups of the moment-angle complex $\mathcal{Z}_{K}$ are determined by the following identity of formal power series:

$$
\prod_{r=1}^{\infty} \frac{\left(1+t^{2 r-1}\right)^{\phi_{2 r}}}{\left(1-t^{2 r}\right)^{\phi_{2 r+1}}}=(1+t)^{-n} \sum_{p, q \geq 0} \operatorname{dim}_{\mathbb{k}} \operatorname{Tor}_{p, q}^{S / I}(\mathbb{k}, \mathbb{k}) t^{q} .
$$

Proof. By work of Notbohm and Ray [43], the space $\mathcal{D} \mathcal{J}(K)$ is known to be formal. Hence, the Eilenberg-Moore spectral sequence of the path-loop fibration of $\mathcal{D} \mathcal{J}(K)$ degenerates at the $E_{2}$ term, see [28]. Together with the MilnorMoore theorem, this implies that the Hopf algebra $U\left(\mathfrak{g}_{\mathcal{D} \mathcal{J}}\right)$ is isomorphic to $\operatorname{Tor}^{H^{*}(\mathcal{D} \mathcal{J}(K), \mathbb{k})}(\mathbb{k}, \mathbb{k})$. Finally, since $H^{*}(\mathcal{D} \mathcal{J}(K), \mathbb{k})=S / I$ (by Theorem 3.1.2) and $\mathbb{k}$ has characteristic zero, we obtain the claimed identity from the PoincaréBirkhoff-Witt theorem, see [42].

Remark 4.1.2. The generating function on the right-hand side of (16) is known to be rational in the case of a Stanley-Reisner ring: see [3]. In the terminology of commutative algebra, the ring $S / I$ is Golod-attached, which means that the canonical projection $S \rightarrow S / I$ factors through a sequence of surjections, each of which is a Golod homomorphism. This in turn means that all the Massey products in a certain DGA vanish; for a systematic discussion, see Avramov [1].

4.2. Flag complexes and Koszul duality. We will give examples later on where both sides of (16) can be computed explicitly. One such general case is that of $K$ a flag complex (meaning, any missing face of $K$ has precisely 2 vertices).

For a quadratic algebra $A=T(V) / J$, let $A^{!}=T\left(V^{*}\right) / J^{\perp}$ denote its quadratic dual. Let $\operatorname{Ext}_{A}(\mathbb{k}, \mathbb{k})$ be the Yoneda algebra, and let $\operatorname{Ext}_{A}^{1}(\mathbb{k}, \mathbb{k})$ be the subalgebra generated by degree 1 elements. As shown by Löfwall [31], $A^{!} \cong \operatorname{Ext}_{A}^{1}(\mathbb{k}, \mathbb{k})$. Recall $A$ is a Koszul algebra if $\operatorname{Ext}_{A}^{1}(\mathbb{k}, \mathbb{k})=\operatorname{Ext}_{A}(\mathbb{k}, \mathbb{k})$; see Fröberg's survey [23] as a general reference. Since $\mathbb{k}$ has characteristic zero, $\operatorname{Ext}_{A}(\mathbb{k}, \mathbb{k})$ is the $\mathbb{k}$-dual of $\operatorname{Tor}^{A}(\mathbb{k}, \mathbb{k})$. 
Theorem 4.2.1. Let $K$ be a flag complex, with Stanley-Reisner ring $S / I$. Then the ranks $\phi_{r}$ of the homotopy groups of the moment-angle complex $\mathcal{Z}_{K}$ are given by:

$$
\prod_{r=1}^{\infty} \frac{\left(1+t^{2 r-1}\right)^{\phi_{2 r}}}{\left(1-t^{2 r}\right)^{\phi_{2 r+1}}}=h(H, i \sqrt{t},-i \sqrt{t})^{-1},
$$

where $H=\operatorname{Tor}^{S}(S / I, \mathbb{k})$ is the cohomology ring of $\mathcal{Z}_{K}$, and $h(H, s, t)$ is its bigraded Hilbert series.

Proof. In this case, the ideal $I$ is quadratic, generated by monomials $x_{i} x_{j}$ for which $\{i, j\} \notin K$. Then $S / I$ is a Koszul algebra, see [23].

Now the "Löfwall formula" for quadratic duals says $h\left(U\left(\mathfrak{g}_{\mathcal{D} \mathcal{J}}\right), t\right)=h(S / I,-t)^{-1}$. On the other hand, a standard Euler characteristic calculation shows

$$
\begin{aligned}
h\left(S / I, t^{2}\right) & =\frac{1}{\left(1-t^{2}\right)^{n}} \sum_{p, q}(-1)^{p} \operatorname{dim}_{\mathbb{k}} \operatorname{Tor}_{p, q}^{S}(S / I, \mathbb{k}) t^{p+q} \\
& =\frac{h(H, t,-t)}{\left(1-t^{2}\right)^{n}}
\end{aligned}
$$

so $h\left(U\left(\mathfrak{g}_{\mathcal{D} \mathcal{J}}\right), t^{2}\right)=\left(1+t^{2}\right)^{-n} h(H, i t,-i t)^{-1}$. The proof is completed by combining this equality with (15).

\section{The Cellular Cochain Algebra and Massey Products}

In this section, we outline Buchstaber and Panov's definition of the cochain algebra of $\mathcal{Z}_{K}$, and Baskakov's setup for computing Massey products in $H^{*}\left(\mathcal{Z}_{K}, \mathbb{k}\right)$. We start with a discussion of formality and Massey products.

5.1. DGA's and formality. Let $\mathbb{k}$ be a field, or the integers. By a differential graded algebra (DGA) we mean a graded $\mathbb{k}$-algebra $A$, endowed with a differential $d: A \rightarrow A$ of degree 1 . The algebra $A$ is said to be (graded)-commutative (CDGA) if $a b=(-1)^{|a||b|} b a$, for every homogeneous elements $a, b \in A$, where $|a|$ denotes the degree of $a$.

Let $A$ be a DGA. We shall assume the cohomology algebra $H^{*}(A)$ is commutative. We can turn $H^{*}(A)$ into a DGA by assigning to it the zero differential. The differential graded algebra $(A, d)$ is said to be formal if there is a sequence of DGA morphisms (going either way), connecting $(A, d)$ to $\left(H^{*}(A), 0\right)$ and inducing isomorphisms in cohomology.

The simplest formality test is provided by the Massey products. As is wellknown, if $(A, d)$ is formal, then all Massey products (of order 3 or higher) vanish; see $[15,52]$. Let us briefly review the relevant definitions; for simplicity, we will only treat the triple products here. 
Assume $\alpha_{1}, \alpha_{2}, \alpha_{3}$ are homogeneous elements in $H^{*}(A)$ such that $\alpha_{1} \alpha_{2}=$ $\alpha_{2} \alpha_{3}=0$. Pick representative cocycles $a_{i}$ for $\alpha_{i}$, and elements $x, y \in A$ such that $d x=a_{1} a_{2}$ and $d y=a_{2} a_{3}$. Setting $\bar{a}=(-1)^{|a|}$, it is readily seen that $x a_{3}-\bar{a}_{1} y$ is a cocyle. The set of cohomology classes of all such cocycles is the Massey triple product $\left\langle\alpha_{1}, \alpha_{2}, \alpha_{3}\right\rangle$. The image of this set in the quotient ring $H^{*}(A) /\left(\alpha_{1}, \alpha_{3}\right)$ is a well-defined element of degree $\left|\alpha_{1}\right|+\left|\alpha_{2}\right|+\left|\alpha_{3}\right|-1$; we say $\left\langle\alpha_{1}, \alpha_{2}, \alpha_{3}\right\rangle$ is non-vanishing if this element is not 0 . The Massey product $\left\langle\alpha_{1}, \alpha_{2}, \alpha_{3}\right\rangle$ is said to be decomposable if it contains a cohomology class that can be written as a product $\lambda \nu$ of two elements in $H^{>0}(A)$; otherwise, it is called indecomposable.

Lemma 5.1.1. Let $(A, d)$ and $\left(A^{\prime}, d^{\prime}\right)$ be two DGA's. Suppose $\rho:\left(A^{\prime}, d^{\prime}\right) \rightarrow$ $(A, d)$ is a surjective chain map, chain homotopic to a ring map, and inducing an isomorphism $\rho^{*}: H^{*}\left(A^{\prime}, d^{\prime}\right) \rightarrow H^{*}(A, d)$. If $\left\langle\alpha_{1}, \alpha_{2}, \alpha_{3}\right\rangle$ is a non-vanishing Massey product in $H^{*}(A, d)$, then $\left(\rho^{*}\right)^{-1}\left\langle\alpha_{1}, \alpha_{2}, \alpha_{3}\right\rangle$ is a non-vanishing Massey product in $H^{*}\left(A^{\prime}, d^{\prime}\right)$.

Proof. Pick representatives as above, so that $\left\langle\alpha_{1}, \alpha_{2}, \alpha_{3}\right\rangle=\left[x a_{3}-\bar{a}_{1} y\right]$. Using the fact that $\rho$ is a surjective quasi-isomorphism, we may find cocycles $a_{1}^{\prime}, a_{2}^{\prime}, a_{3}^{\prime} \in A^{\prime}$ such that $\rho\left(a_{i}^{\prime}\right)=a_{i}$, and cochains $x^{\prime}, y^{\prime} \in A^{\prime}$ such that $d x^{\prime}=a_{1}^{\prime} a_{2}^{\prime}$ and $d y^{\prime}=a_{2}^{\prime} a_{3}^{\prime}$, and thus form the Massey product $\left\langle\alpha_{1}^{\prime}, \alpha_{2}^{\prime}, \alpha_{3}^{\prime}\right\rangle=\left[x^{\prime} a_{3}^{\prime}-\bar{a}_{1}^{\prime} y^{\prime}\right]$.

Now, by assumption, there is a a degree 1 map $\sigma:\left(A^{\prime}, d^{\prime}\right) \rightarrow(A, d)$ so that $\rho\left(a^{\prime} b^{\prime}\right)-\rho\left(a^{\prime}\right) \rho\left(b^{\prime}\right)=\left(\sigma d^{\prime}+d \sigma\right)\left(a^{\prime} b^{\prime}\right)$, for every $a^{\prime}, b^{\prime} \in A^{\prime}$. This implies $\rho\left(x^{\prime} a_{3}^{\prime}-\right.$ $\left.\bar{a}_{1}^{\prime} y^{\prime}\right)-\left(x a_{3}-\bar{a}_{1} y\right) \in \operatorname{im}(d)$, finishing the proof.

5.2. Formal spaces. To a space $X$, Sullivan associates in [52] a commutative DGA: the rational algebra of polynomial forms, $\left(A_{\mathrm{PL}}(X ; \mathbb{Q}), d\right)$. The space $X$ is said to be rationally formal (or simply, formal) if $\left(A_{\mathrm{PL}}(X ; \mathbb{Q}), d\right)$ is formal in the category of CDGA's. For more details, and equivalent definitions, see [15, 20].

Examples of formal spaces include spheres; Eilenberg-MacLane spaces $K(\pi, n)$ with $n>1$; compact, connected Lie groups $G$ and their classifying spaces $B G$ [52]; and compact Kähler manifolds [15]. Formality is preserved under wedges and products of spaces, and connected sums of manifolds.

We say $X$ is integrally formal if the singular cochain algebra $\left(C^{*}(X, \mathbb{Z}), d\right)$ is formal. From the definition it is apparent that, if $\left(C^{*}(X, \mathbb{Z}), d\right)$ is formal, so is $\left(C^{*}(X, \mathbb{k}), d\right)$, for any choice of field $\mathbb{k}$ of characteristic 0 . A result of Watkiss, recorded in [20, Corollary 10.10], implies that if $X$ is rationally formal, then $\left(C^{*}(X, \mathbb{k}), d\right)$ is formal, where $\mathbb{k}$ is any field of characteristic zero.

In what follows, we will show that, for certain spaces $X$, the singular cochain DGA $\left(C^{*}(X, \mathbb{Q}), d\right)$ is not formal. By the logic above, then, $X$ is neither integrally formal, nor rationally formal. Since the notions coincide, we will simply write that $X$ is not formal. 
In [43], Notbohm and Ray show that the Davis-Januszkiewicz spaces, $\mathcal{D} \mathcal{J}(K)=$ $\mathcal{Z}_{K}\left(B S^{1}\right)$, are both integrally formal and rationally formal. It is also known that the classifying spaces for right-angled Artin groups, $K\left(G_{\Gamma}, 1\right)=\mathcal{Z}_{\Delta(\Gamma)}\left(S^{1}\right)$, are rationally formal; see [48].

If $X$ is formal, and $\left\langle\alpha_{1}, \alpha_{2}, \alpha_{3}\right\rangle$ is a Massey product computed from $\left(C^{*}(X, \mathbb{k}), d\right)$, then $\left\langle\alpha_{1}, \alpha_{2}, \alpha_{3}\right\rangle$ vanishes. Thus, non-vanishing Massey products provide a handy tool for detecting non-formality.

As shown by Stasheff [50], if $X$ is a $k$-connected CW-complex of dimension $n \leq 3 k+1$, then $X$ is formal. This is best possible: attaching a cell $e^{3 k+2}$ to the wedge $S^{k+1} \vee S^{k+1}$ via the iterated Whitehead product $\left[\iota_{1},\left[\iota_{1}, \iota_{2}\right]\right]$ yields a non-formal CW-complex. According to Miller [41], the dimension bound can be relaxed for manifolds: if $X$ is a compact, $k$-connected manifold of dimension $n \leq 4 k+2$, then $X$ is formal. Again, this bound is best possible, see [21], [17].

5.3. The cellular cochain algebra of $\mathcal{Z}_{K}$. Given a CW-complex $X$, let $\left(C_{\mathrm{cw}}^{*}(X), d\right)$ be its (integral) cellular cochain complex. The cellular cup-product map may be defined as the composite

$$
\cup: C_{\mathrm{cw}}^{*}(X) \otimes C_{\mathrm{cw}}^{*}(X) \stackrel{\times}{\longrightarrow} C_{\mathrm{cw}}^{*}(X \times X) \stackrel{\widetilde{\Delta}}{\longrightarrow} C_{\mathrm{cw}}^{*}(X),
$$

where $\widetilde{\Delta}$ is a cellular approximation to the diagonal map $\Delta: X \rightarrow X \times X$. Unfortunately, such an approximation is not functorial, and the cup-product map on $C_{\mathrm{cw}}^{*}(X)$ is not associative, in general-although, of course, $\cup$ induces an associative and commutative product on $H^{*}(X, \mathbb{Z})$.

Despite these difficulties, Buchstaber, Panov, and Baskakov $[9,6,44]$ were able to define a DGA structure on the cellular cochain complex of a moment-angle complex $\mathcal{Z}_{K}=\mathcal{Z}_{K}\left(D^{2}, S^{1}\right)$, over a field $\mathbb{k}$. Let us review their construction.

The unit disk $D^{2}=\{z \in \mathbb{C}|| z \mid \leq 1\}$ comes endowed with a natural cell structure: $D^{2}=e^{0} \cup e^{1} \cup e^{2}$, with 0 -skeleton the point $z=1$, with 1-skeleton the boundary circle $S^{1}$, and with 2 -skeleton the disk itself. The diagonal map of $D^{2}$ has a natural cellular approximation, $\widetilde{\Delta}: D^{2} \rightarrow D^{2} \times D^{2}$, sending $z=r e^{i \theta}$ to $\left(1+r\left(e^{2 i \theta}-1\right), 1\right)$ for $0 \leq \theta<\pi$ and to $\left(1,1+r\left(e^{2 i \theta}-1\right)\right)$ for $\pi \leq \theta<2 \pi$. With the cup product defined by $\widetilde{\Delta}$, the cellular cochain complex $C_{\mathrm{cw}}^{*}\left(D^{2}\right)$ becomes a commutative DGA, with generators $u$ and $x$ dual to $e^{1}$ and $e^{2}$, multiplication $x^{2}=x u=0$, and differential $d u=x, d x=0$.

Now let $K$ be a simplicial complex on $[n]$, and let $\mathcal{Z}_{K} \subset\left(D^{2}\right)^{\times n}$ be the corresponding moment-angle complex. The diagonal map of $\left(D^{2}\right)^{\times n}$ has cellular approximation $\widetilde{\Delta}^{\times n}$; this map restricts to a cellular approximation $\widetilde{\Delta}_{K}$ to the diagonal map of $\mathcal{Z}_{K}$. The resulting cup product on the cellular cochain complex 
of $\mathcal{Z}_{K}$, with coefficients in $\mathbb{k}$, yields a commutative DGA with presentation

$$
C_{\mathrm{cw}}^{*}\left(\mathcal{Z}_{K}, \mathbb{k}\right)=\left(\left(S / I_{K}\right) \otimes_{\mathbb{k}} E\right) /\left(x_{i}^{2}=x_{i} u_{i}=0\right),
$$

where $S=\mathbb{k}\left[x_{1}, \ldots, x_{n}\right]$ is the polynomial ring with generators $x_{i}$ in degree $(0,2), E=\bigwedge\left[x_{1}, \ldots, x_{n}\right]$ is the exterior algebra with generators $u_{i}$ in degree $(1,1)$, and with differential $d$ given by $d u_{i}=x_{i}, d x_{i}=0$. Additively, this algebra is generated by square-free monomials $x_{\sigma} u_{\mathcal{I}}$, for all choices of $\sigma \in K$ and $\mathcal{I} \subseteq[n]$ for which $\sigma \cap \mathcal{I}=\emptyset$. The construction has the following naturality property: If $f: K \rightarrow L$ is a simplicial map, then $\mathcal{Z}_{f}: \mathcal{Z}_{K} \rightarrow \mathcal{Z}_{L}$ commutes with the diagonal approximations, and so $\mathcal{Z}_{f}^{*}: C_{\mathrm{cw}}^{*}\left(\mathcal{Z}_{L}, \mathbb{k}\right) \rightarrow C_{\mathrm{cw}}^{*}\left(\mathcal{Z}_{K}, \mathbb{k}\right)$ is a DGA morphism.

Baskakov, Buchstaber and Panov [6] show that the natural surjection from the Koszul complex of the Stanley-Reisner ring to the cellular cochain algebra,

$$
\pi: S / I_{K} \otimes_{\mathbb{k}} E \rightarrow C_{\mathrm{cw}}^{*}\left(\mathcal{Z}_{K}, \mathbb{k}\right),
$$

is a (bigraded) quasi-isomorphism of DGAs. This recovers the isomorphism $H^{q}\left(\mathcal{Z}_{K}, \mathbb{k}\right)_{p} \cong \operatorname{Tor}_{p, q}^{S}\left(S / I_{K}, \mathbb{k}\right)$ from $(10)$, grading Tor by homological and internal degree, respectively.

5.4. Multiplicative structures in resolutions. Formality of the momentangle complex $\mathcal{Z}_{K}$ has a quite closely related notion in commutative algebra.

Given a $\mathbb{k}$-algebra homomorphism $S \rightarrow S / I$, a projective resolution $P$ of $S / I$ over $S$ is said to have multiplicative structure if $P$ has the structure of a commutative DGA, and the surjection $P \rightarrow S / I$ is a quasi-isomorphism of DGAs over $S$ (for details, see Avramov's survey [2]). Also recall that graded modules over commutative graded rings have minimal free resolutions: that is, resolutions in which the differential can be expressed as a matrix whose entries have strictly positive degree. Such resolutions are essentially unique.

Proposition 5.4.1. Suppose the minimal free resolution $P$ of $S / I$ over $S$ has multiplicative structure. Then $C_{\mathrm{cw}}^{*}\left(\mathcal{Z}_{K}, \mathbb{k}\right)$ is formal.

Proof. The Koszul complex $S \otimes_{\mathbb{k}} E$ is a DGA resolution of $\mathbb{k}$. Since $\operatorname{Tor}^{S}(S / I, \mathbb{k})$ may be computed by resolving either $S / I$ or $\mathbb{k}$, the augmentation maps in each resolution give quasi-isomorphisms of DGAs (see $[2,2.3 .2]$ ):

$$
P \otimes_{S} \mathbb{k} \leftarrow P \otimes_{S} E \rightarrow S / I \otimes_{S} E,
$$

each having homology $\operatorname{Tor}^{S}(S / I, \mathbb{k})=H^{*}\left(\mathcal{Z}_{K}, \mathbb{k}\right)$. Since $P$ is minimal, however, $P \otimes_{S} \mathbb{k}$ also has trivial differential, so $P \otimes_{S} \mathbb{k} \cong H^{*}\left(\mathcal{Z}_{K}, \mathbb{k}\right)$, which means that each DGA in (22) is formal. By $(21), C_{\mathrm{cw}}^{*}\left(\mathcal{Z}_{K}, \mathbb{k}\right)$ is formal. 
5.5. The Baskakov formula. Using the cellular cochain algebra model for $\mathcal{Z}_{K}$, Baskakov [4] gave an explicit formula for the cup product in $H^{*}\left(\mathcal{Z}_{K}, \mathbb{Z}\right)$, in terms of pairings between full subcomplexes. Let us recall Baskakov's formula (see also Panov [44]) by means of (20).

For each subset $\mathcal{I} \subseteq[n]$, let $K_{\mathcal{I}}$ denote the full subcomplex of $K$ on vertices $\mathcal{I}$. For each $\sigma \in K_{\mathcal{I}}$, let $s=|\sigma|$ and $p=|\mathcal{I}|$. Then define a map to the (reduced) group of simplicial cochains on $K_{\mathcal{I}}$,

$$
\phi_{\mathcal{I}}: C_{\mathrm{cw}}^{p, p+2 s}\left(\mathcal{Z}_{K}\right) \rightarrow \widetilde{C}^{s-1}\left(K_{\mathcal{I}}\right),
$$

by letting $\phi_{\mathcal{I}}\left(x_{\sigma} u_{\mathcal{I}-\sigma}\right)=\chi_{\sigma}$, where $\chi_{\sigma}$ denotes the indicator function on the simplex $\sigma$ in $K_{\mathcal{I}}$. (By convention, $\widetilde{C}^{-1}(\emptyset)=\mathbb{k}$.) The maps $\left\{\phi_{\mathcal{I}}\right\}$ assemble to give an isomorphism of cochain complexes,

$$
\Phi: C_{\mathrm{cw}}^{*}\left(\mathcal{Z}_{K}\right) \rightarrow \bigoplus_{\mathcal{I} \subseteq[n]} \widetilde{C}^{*}\left(K_{\mathcal{I}}\right)
$$

The map $\Phi$ is, in fact, a ring isomorphism, where the multiplication on the left is defined by (20), while the multiplication on the right is defined as follows. For $\alpha \in \widetilde{C}^{p}\left(K_{\mathcal{I}}\right)$ and $\alpha^{\prime} \in \widetilde{C}^{p^{\prime}}\left(K_{\mathcal{I}^{\prime}}\right)$, the product $\alpha \cdot \alpha^{\prime} \in \widetilde{C}^{p+p^{\prime}+1}\left(K_{\mathcal{I} \sqcup \mathcal{I}^{\prime}}\right)$ is given by

$$
\alpha \cdot \alpha^{\prime}= \begin{cases}0, & \text { if } \mathcal{I} \cap \mathcal{I}^{\prime} \neq \emptyset, \\ j^{*} \psi\left(\alpha \otimes \alpha^{\prime}\right), & \text { if } \mathcal{I} \cap \mathcal{I}^{\prime}=\emptyset,\end{cases}
$$

where $j: K_{\mathcal{I} \sqcup \mathcal{I}^{\prime}}=K_{\mathcal{I}} \sqcup K_{\mathcal{I}^{\prime}} \hookrightarrow K_{\mathcal{I}} * K_{\mathcal{I}^{\prime}}$ is the inclusion into the simplicial join, and $\psi: \widetilde{C}^{p}\left(K_{\mathcal{I}}\right) \otimes \widetilde{C}^{p^{\prime}}\left(K_{\mathcal{I}^{\prime}}\right) \stackrel{\cong}{\longrightarrow} \widetilde{C}^{p+p^{\prime}+1}\left(K_{\mathcal{I}} * K_{\mathcal{I}^{\prime}}\right)$ is the standard isomorphism between the respective reduced simplicial cochain complexes.

Taking cohomology, one obtains the (additive) formula of Hochster [29],

$$
\operatorname{Tor}_{p-s, p+s}^{S}(S / I, \mathbb{Z})=\bigoplus_{\substack{I \subseteq[n] \\|I|=p}} \widetilde{H}^{s-1}\left(K_{\mathcal{I}}, \mathbb{Z}\right)
$$

5.6. Massey products in $\mathcal{Z}_{K}$. Let us now show that the non-formality of $\mathcal{Z}_{K}$ can be detected by non-vanishing Massey products - not just in the singular cochain algebra $C^{*}\left(\mathcal{Z}_{K}, \mathbb{k}\right)$, but also in the much simpler (and commutative) cellular cochain algebra $C_{\mathrm{cw}}^{*}\left(\mathcal{Z}_{K}, \mathbb{k}\right)$.

Proposition 5.6.1. Suppose the differential graded algebra $C_{\mathrm{cw}}^{*}\left(\mathcal{Z}_{K}, \mathbb{k}\right)$ carries a non-vanishing triple Massey product. Then $\mathcal{Z}_{K}$ is not formal.

Proof. Simplicial approximation yields a surjective homomorphism $\rho: C^{*}\left(\left(D^{2}\right)^{\times n}\right.$, $\mathbb{k}) \rightarrow C_{\mathrm{cw}}^{*}\left(\left(D^{2}\right)^{\times n}, \mathbb{k}\right)$, commuting with the differentials. Since $\left(D^{2}\right)^{\times n}$ is acyclic, 

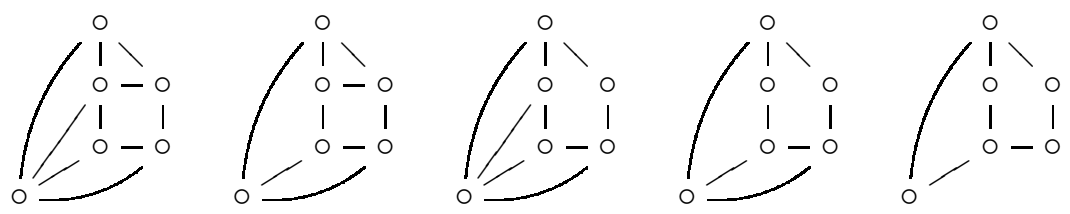

Figure 2. The five obstruction graphs

$\rho(a b)-\rho(a) \rho(b)$ is a cellular coboundary, for any singular cochains $a$, $b$; thus, $\rho$ is also a ring map, up to chain homotopy. Now consider the commuting diagram

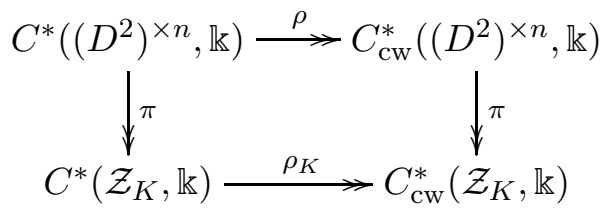

where the vertical arrows are the canonical projections (both morphisms of DGA's), while $\rho_{K}$ is the restriction of $\rho$. Chasing the diagram, we see that the chain map $\rho_{K}$ is also a ring map, up to chain homotopy. The conclusion follows from Lemma 5.1 .1 and the standard Massey product formality test for $C^{*}\left(\mathcal{Z}_{K}, \mathbb{k}\right)$.

\section{TRiple Massey products in LOWEst Degree}

In [5], Baskakov constructs a family of simplicial complexes $K$ for which the cellular cochain algebra defined above has non-vanishing Massey triple products: a complete discussion is found in [44]. Here, we show that one can characterize the complexes $K$ for which $H^{*}\left(\mathcal{Z}_{K}, \mathbb{k}\right)$ contains a non-trivial Massey product in lowest possible degree.

6.1. The five obstruction graphs. Let $K$ be a simplicial complex on $n$ vertices. Let $K^{(1)}$ denote the 1-skeleton of $K$.

Theorem 6.1.1. The following are equivalent:

(i) There exist cohomology classes $\alpha, \beta, \gamma \in H^{3}\left(\mathcal{Z}_{K}, \mathbb{k}\right)$ for which $\langle\alpha, \beta, \gamma\rangle$ is defined and non-trivial.

(ii) The underlying graph of $K^{(1)}$ contains an induced subgraph isomorphic to one of the five graphs in Figure 2.

Moreover, all Massey products arising in this fashion are decomposable.

By the functoriality of the moment-angle construction on inclusions of full subcomplexes $L \hookrightarrow K$, (Lemma 2.2.3 and subsection 5.3), the Theorem is implied by the following statement: 
Proposition 6.1.2. If $L$ is a simplicial complex on six vertices, $H^{*}\left(\mathcal{Z}_{L}, \mathbb{k}\right)$ has a non-trivial Massey triple product if and only if its 1-skeleton is shown in Figure 2. Moreover, any non-trivial Massey product in $H^{*}\left(\mathcal{Z}_{L}, \mathbb{k}\right)$ is decomposable.

The proof of this Proposition will occupy the rest of this section.

6.2. Proof of " $\Leftarrow$ " of Proposition 6.1.2: Suppose the one-skeleton of $L$ is one of Figure 2. None of these graphs contains a complete subgraph on four vertices, from which it follows $L$ is a 2-complex. The only two-cells which may appear are the interiors of the triangles. Regardless of the two-cells, $\widetilde{H}^{p}(L, \mathbb{k}) \neq 0$ iff $p=1$.

Label the vertices of the "obstruction" graphs as in Figure 3. Then $L^{(1)}$ is obtained by omitting any subset of the dotted edges. Recall $L_{\mathcal{I}}$ denotes the full subcomplex on index set $\mathcal{I}$; we will slightly abuse notation, and write, e.g., $L_{i, j}$ or even $L_{i j}$ for $L_{\{i, j\}}$.

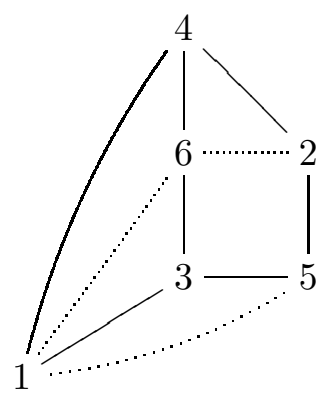

Figure 3. A labeled obstruction graph

Let $\alpha, \beta$, and $\gamma$ be non-trivial classes in $\widetilde{H}^{0}\left(L_{i, i+1}, \mathbb{k}\right)$, for $i=1,3,5$, respectively. Abusing notation, we shall identify $\alpha, \beta$, and $\gamma$ with their images in $H^{3}\left(\mathcal{Z}_{L}, \mathbb{k}\right)$ under the isomorphism $\Phi_{*}$ induced by the chain map (24). Then, by means of Baskakov's formula (25), we show the triple product $\langle\alpha, \beta, \gamma\rangle \in$ $H^{8}\left(\mathcal{Z}_{L}, \mathbb{k}\right)$ is non-trivial, as follows.

We will work with cellular cochains and denote cocycles by indicator functions on simplicial subcomplexes. It should be noted that the underlying subcomplex is part of the data, but for legibility reasons will be taken to be implicit in what follows.

Since Massey products are linear in each argument, we may replace each cocycle with a nonzero scalar multiple without loss of generality. Accordingly, let $\alpha=$ $\left[\chi_{2}\right], \beta=\left[\chi_{3}\right]$, and $\gamma=\left[\chi_{5}\right]$. From Figure 3, we see the subcomplexes $L_{1234}$ and $L_{3456}$ are both paths: in particular

$$
\widetilde{H}^{p}\left(L_{1234}, \mathbb{k}\right)=\widetilde{H}^{p}\left(L_{3456}, \mathbb{k}\right)=0 \text { for all } p,
$$


so $\alpha \beta=\beta \gamma=0$.

Then, multiplying cochains with the Baskakov construction, we see $\chi_{2} \chi_{3}=0$ while $\chi_{3} \chi_{5}=\chi_{35}=-\delta\left(\chi_{5}\right)$. It follows that the triple product is represented by the cochain

$$
0 \cdot \chi_{5}-(-1)^{3} \chi_{2} \cdot\left(-\chi_{5}\right)=-\chi_{25} .
$$

Let $\omega=\left[-\chi_{25}\right]$. It is easily seen that $\omega$ is nonzero in $H^{1}\left(L_{123456}, \mathbb{k}\right)=H^{8}\left(\mathcal{Z}_{L}, \mathbb{k}\right)$, regardless of the filling of the two-cells.

Last, we must check that the Massey product $\langle\alpha, \beta, \gamma\rangle$ is non-trivial with respect to indeterminacy; that is, $\omega$ does not lie in the ideal of $H^{*}\left(\mathcal{Z}_{L}, \mathbb{k}\right)$ generated by $\{\alpha, \gamma\}$. For this, suppose $\alpha \eta \in H^{8}\left(\mathcal{Z}_{L}, \mathbb{k}\right)$ is supported on $L_{123456}$. For grading reasons, the support of $\eta$ is $L_{3456}$. However, from (28), $\eta$ must be zero. The same argument applies to $\gamma$, so $(\alpha, \gamma) \cap H^{8}\left(\mathcal{Z}_{L}, \mathbb{k}\right)=0$; the indeterminacy for this Massey product is zero.

Finally, we claim that $\omega=\nu \nu^{\prime}$ for certain classes $\nu, \nu^{\prime} \in H^{4}\left(\mathcal{Z}_{L}, \mathbb{k}\right)$; that is, $\omega$ is decomposable. Let $\nu=\left[-\chi_{2}\right]$ on $L_{123}$, a generator of $\widetilde{H}^{0}\left(L_{123}, \mathbb{k}\right)$. Similarly, let $\nu^{\prime}=\left[\chi_{5}\right]$ on $L_{456}$. Since $\omega=\left[-\chi_{25}\right]$, the claim follows by $(25)$.

6.3. Proof of " $\Rightarrow$ " of Proposition 6.1.2: Suppose that $L$ is a simplicial complex on six vertices, and it possesses a non-trivial Massey product $\langle\alpha, \beta, \gamma\rangle$. For grading reasons, each of $\alpha, \beta, \gamma$ must be supported on pairwise-disjoint, 2-vertex, disconnected subcomplexes. Without loss of generality, suppose $\alpha, \beta, \gamma$ are supported on vertices $S_{1}=\{1,2\}, S_{2}=\{3,4\}$, and $S_{3}=\{5,6\}$, respectively.

Let the graph $G$ be the edge-complement of $L^{(1)}$. We shall show that $G$ must be obtained from the graph below by adding any subset of the dotted edges. Then, by comparing with Figure 3, the implication is proven.

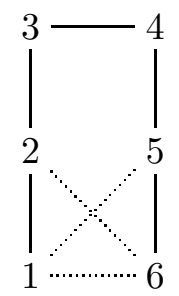

Immediately, $G$ contains edges $i, i+1$ for $i=1,3,5$.

Lemma 6.3.1. $G$ contains edges joining $S_{i}$ to $S_{i+1}$ for $i=1,2$.

Proof. Since $\alpha, \beta, \gamma$ define a Massey product, $\alpha \beta=0$. It follows that $L_{1234}$ is not a cycle, so there is at least one edge from $S_{1}$ to $S_{2}$. A symmetric argument applies to $S_{2}, S_{3}$. 
Lemma 6.3.2. $G$ does not contain a path $v_{1} v_{2} v_{3}$, where $v_{i} \in S_{i}$ for $i=1,2,3$.

Proof. Suppose it did. Then, up to nonzero scalar multiples, $\alpha=\left[\chi_{v_{1}}\right], \beta=\left[\chi_{v_{2}}\right]$, and $\gamma=\left[\chi_{v_{3}}\right]$. The edge $v_{i} v_{i+1}$ in $G$ means there is no edge between $v_{i}$ and $v_{i+1}$ in $L$, so $\chi_{v_{i}} \chi_{v_{i+1}}=0$ for $i=1,2$. Then the Massey product vanishes, contradicting the hypotheses.

Up to relabeling, then, $G$ contains the path $12 \cdots 6$, and vertices 3,4 both have degree 2 . It remains to check the following.

Lemma 6.3.3. $G$ does not contain the edge 25 .

Proof. If it did, represent $\alpha, \beta, \gamma$ by $\chi_{2}, \chi_{3}, \chi_{5}$, respectively. Then $\chi_{2} \chi_{3}=0$, while $\chi_{3} \chi_{5}=-\delta\left(\chi_{5}\right)$. Then an edge $v_{2} v_{5}$ would make $\chi_{2} \chi_{5}=0$ and the Massey product cohomologous to zero, a contradiction.

The proof of Proposition 6.1.2 now follows directly from the previous three Lemmas. The Massey product $\langle\alpha, \beta, \gamma\rangle$ is, up to a nonzero scalar, the one constructed in 6.3 ; in particular, it is decomposable.

\section{Formal MOMENT-ANGLE MANIFOLDS}

In this and the next section, we consider closed manifolds obtained from sphere triangulations by means of the moment-angle construction. We begin with the formal ones.

7.1. Moment-angle manifolds. Suppose $K$ is an $n$-vertex triangulation of the sphere $S^{\ell}$. Then, as shown by Buchstaber and Panov [9], the moment-angle complex $\mathcal{Z}_{K}=\mathcal{Z}_{K}\left(D^{2}, S^{1}\right)$ is a smooth, compact, connected manifold of dimension $n+\ell+1$; moreover, $\mathcal{Z}_{K}$ is 2 -connected, and in fact, $2 k$-connected, if $K$ is $k$-neighborly.

Some moment-angle manifolds can be described in simple terms. As noted in Example 2.1.3, if $K$ is the boundary of the $(n-1)$-simplex, then $\mathcal{Z}_{K}=S^{2 n-1}$. Also, if $K=\left(S^{0}\right)^{* d}$ (the simplicial join of $d$ copies of $\left.S^{0}\right)$ is the $(d-1)$-dimensional hyperoctahedron, then $\mathcal{Z}_{K}=\left(S^{3}\right)^{\times d}$, from Corollary 2.1.4. In general, though, moment-angle manifolds can exhibit quite a complicated structure, both from the point of view of their cohomology ring and their Massey products.

We start with an observation concerning the formality of such manifolds.

Proposition 7.1.1. Suppose $K$ is an $n$-vertex triangulation of $S^{\ell}$.

(i) If $n+\ell \leq 9$, then $\mathcal{Z}_{K}$ is formal.

(ii) If $K$ is $k$-neighborly, and $n+\ell \leq 8 k+1$, then $\mathcal{Z}_{K}$ is formal. 
Proof. Use Miller's result [41], as recounted in $§ 5.2$.

In particular, all triangulations of $S^{2}$ on at most 7 vertices (there are precisely 9 such) yield formal moment-angle manifolds.

7.2. Corner-cutting. We now describe an operation on simplicial complexes which proves to be useful in this context.

Given a simplicial complex $K$ and a maximal simplex $F=\left(v_{0}, v_{1}, \ldots, v_{k}\right)$ in $K$, let $K *_{F} w$ denote the complex obtained by adding a new vertex $w$, removing the simplex $F$, and adding in $k+1$ maximal simplices $\left(v_{0}, v_{1}, \ldots, v_{i-1}, w, v_{i+1}, \ldots, v_{k}\right)$ for $0 \leq i \leq k$.

Note that this operation is dual to cutting the corner from a polytope: if $K$ triangulates $S^{k}$ and $F$ is a maximal simplex of $K$, then let $P$ and $P^{\prime}$ denote the dual polytopes to $K$ and $K *_{F} w$, respectively. Maximal simplices of the triangulation are in bijection with vertices of the dual polytope. So $P^{\prime}$ is obtained from $P$ by cutting off the vertex labeled by $F$ in $P$.

Work of McGavran [36, Theorem 3.4] and Bosio and Meersseman [8, Theorem 6.3] gives the following: if $K$ is obtained from the boundary of a simplex by a sequence of moves as above, then $\mathcal{Z}_{K}$ is diffeomorphic to

$$
\#_{i=1}^{p} S^{a_{i}} \times S^{n-a_{i}}
$$

a connected sum of products of spheres. Consequently, such moment-angle manifolds are formal spaces, and the cohomology ring $H^{*}\left(\mathcal{Z}_{K}, \mathbb{Z}\right)$ has a very simple structure, essentially dictated by the Betti numbers and Poincaré duality.

Example 7.2.1. Denote by $\mathcal{Z}_{n}$ the $(n+2)$-dimensional moment-angle manifold corresponding to an $n$-gon, $n \geq 3$. From the discussion in $\S 2.1$, we know that $\mathcal{Z}_{3}=S^{5}$ and $\mathcal{Z}_{4}=S^{3} \times S^{3}$. More generally, by [36] we have:

$$
\mathcal{Z}_{n}=\#_{j=1}^{n-3} j\left(\begin{array}{c}
n-2 \\
j+1
\end{array}\right) S^{j+2} \times S^{n-j}
$$

for all $n \geq 4$. Computing Betti numbers from this decomposition, we find

$$
b_{k}\left(\mathcal{Z}_{n}\right)=\frac{k(k-2)(n-k)}{(n-1)(n-k+1)} \cdot\left(\begin{array}{l}
n \\
k
\end{array}\right),
$$

for $3 \leq k \leq n-1$, which recovers a result of Buchstaber and Panov [9, Example 4.3.5].

Now, for all $n \geq 4$, the $n$-gon is a flag complex, so Theorem 4.2.1 applies to describe the ranks of the homotopy groups of $\mathcal{Z}_{n}$. We will do so directly. Using a result of Stanley [49], the Hilbert series of $S / I$ is given by the number of faces of each dimension in the $n$-gon:

$$
h(S / I, t)=1+\frac{n t}{1-t}+\frac{n t^{2}}{(1-t)^{2}} .
$$




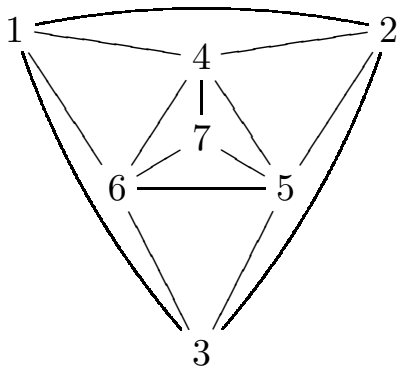

FIgURE 4. A triangulation $K$ of $S^{2}$ for which $\mathcal{Z}_{K}$ is not a connected sum of products of spheres

Then, by (18),

$$
\begin{aligned}
h(H, t,-t) & =\left(1-t^{2}\right)^{n} h\left(S / I, t^{2}\right) \\
& =\left(1-t^{2}\right)^{n-2}\left(1+(n-2) t^{2}+t^{4}\right),
\end{aligned}
$$

where we recall $h(H, s, t)=\sum_{p, q} \operatorname{dim}_{\mathbb{k}} \operatorname{Tor}_{p, q}^{S}(S / I, \mathbb{k}) t^{p} s^{q}$. Using (17),

$$
\prod_{r=1}^{\infty} \frac{\left(1-t^{2 r}\right)^{\phi_{2 r+1}}}{\left(1+t^{2 r+1}\right)^{\phi_{2 r+2}}}=(1+t)^{n-2}\left(1+(2-n) t+t^{2}\right),
$$

from which the ranks of the homotopy groups may be computed recursively. For example,

$$
\begin{aligned}
& \phi_{3}=n(n-3) / 2 \\
& \phi_{4}=n(n-2)(n-4) / 3 \\
& \phi_{5}=n(n-1)(n-3)(n-4) / 4 \\
& \phi_{6}=n(n-1)(n-2)(n-3)(n-4) / 5 \\
& \phi_{7}=n(n-2)(n-3)(n-4)\left(n^{2}-3 n+1\right) / 6,
\end{aligned}
$$

for $n \geq 4$.

7.3. Beyond connected sums of products of spheres. It turns out that not all moment-angle manifolds are of the form (31). The simplest example of this sort, due to Bosio and Meersseman, is presented next.

Example 7.3.1. Let $K$ be the triangulation obtained by adding a vertex to the face of an octahedron, as shown in Figure 4. The non-edges in $K$ are 15, 17, 26, 27,34 , and 37 . The reader may check that the only pairs of non-edges whose union forms a cycle, then, are any choice of two of $\{15,26,34\}$. The manifold $\mathcal{Z}_{K}$ has bigraded Betti numbers as indicated in the following diagram, produced by the software package Macaulay 2 of Grayson and Stillman [26]: 


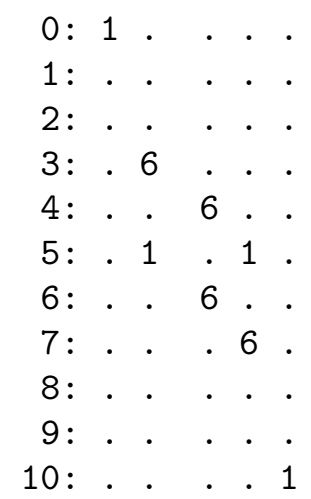

If we denote by $\alpha, \beta$, and $\gamma$, respectively, cohomology classes in $H^{3}\left(\mathcal{Z}_{K}\right)$ supported on edges 15,26 , and 34 in the sense of (24), the products $\alpha \beta, \beta \gamma$, and $\gamma \alpha$ are each nonzero, while all other products of degree-3 classes are zero. In [8, Example 11.5], Bosio and Meersseman observe that this manifold $\mathcal{Z}_{K}$ cannot be even homotopyequivalent to a manifold of the form (31), since the cohomology ring of the latter will not have nonzero cup products of this form.

\section{NON-FORMAL MOMENT-ANGLE MANIFOLDS}

We now turn to moment-angle manifolds that carry non-vanishing Massey products. We will work over a field $\mathbb{k}$ of characteristic 0 .

8.1. An 8-vertex triangulation of $S^{2}$. Since the graphs from Figure 2 are planar, they can be completed to triangulations of the 2 -sphere. These triangulations can be used to produce non-formal moment-angle manifolds.

Example 8.1.1. Let $K$ be the flag complex depicted in Figure 5. The corresponding 11-dimensional manifold $\mathcal{Z}_{K}$ has bigraded Betti numbers as listed below.

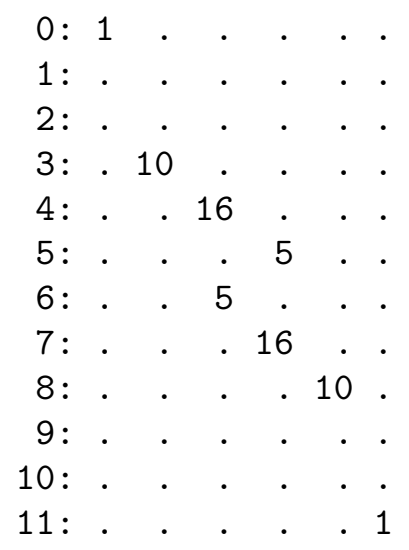




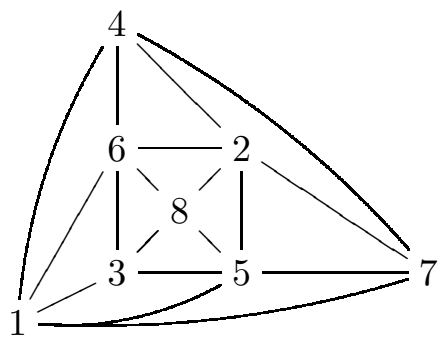

Figure 5. A triangulation $K$ of $S^{2}$ for which $\mathcal{Z}_{K}$ is not formal

By Theorem 6.1.1, there are classes $\alpha, \beta, \gamma \in H^{3}\left(\mathcal{Z}_{K}, \mathbb{k}\right)$ such that $\langle\alpha, \beta, \gamma\rangle \in$ $H^{8}\left(\mathcal{Z}_{K}, \mathbb{k}\right)$ is a non-trivial (decomposable) Massey product, with zero indeterminacy.

Remark 8.1.2. The manifold $\mathcal{Z}_{K}$ from Example 8.1 .1 belongs to an infinite family of non-formal moment-angle manifolds constructed by Baskakov [5]. This is the only member of that family for which $K$ is a triangulation of $S^{2}$.

Remark 8.1.3. This example is minimal: as noted in Proposition 7.1.1, all triangulations of $S^{2}$ on less than 8 vertices yield formal moment-angle manifolds. Moreover, of the 14 distinct 8-point triangulations of $S^{2}$ (as generated by McKay's software plantri [37]), the flag complex from Example 8.1.1 is the unique triangulation $K$ for which $\mathcal{Z}_{K}$ possesses non-trivial Massey products.

8.2. An infinite family. The next result will permit us to construct many more examples of non-formal moment-angle manifolds, not covered by Baskakov's method.

Proposition 8.2.1. If $\mathcal{Z}_{K}$ has a non-trivial triple Massey product, then so does $\mathcal{Z}_{K *_{F} w}$, for any maximal face $F$ of dimension at least 2.

Conversely, if $K$ is a triangulation of $S^{2}, F$ is a triangle, and $\mathcal{Z}_{K *_{F} w}$ has a non-trivial Massey triple product, then so does $\mathcal{Z}_{K}$.

Proof. By assuming $F$ has dimension at least two, the one-skeleton of $K_{123456}$ is unchanged. By the work above, the non-triviality of the Massey product depends only on this one-skeleton.

To prove the converse, it is enough to show that $w$ cannot be a vertex $\{1, \ldots, 6\}$ of an excluded subcomplex as in Figure 3 ; then $K$ and $K *_{F} w$ will have the same subcomplex on $\{1, \ldots, 6\}$, hence the same non-trivial Massey product.

Consider completing any of the five graphs in Figure 2 to a triangulation. Any vertex on a face with more than three edges, including the face at infinity, must be joined to a vertex that subdivides that face. So, by inspection, each of $1, \ldots, 6$ 
must have degree at least 4 in the 1-skeleton of any triangulation of $S^{2}$. By hypothesis, $w$ has degree 3 , so $w \neq i$ for each $i$. This completes the proof.

Using Example 8.1.1 and Proposition 8.2.1, we obtain the following.

Corollary 8.2.2. There are infinitely many triangulations $K$ of $S^{2}$ for which the moment-angle manifold $\mathcal{Z}_{K}$ has non-trivial triple Massey products, and thus is not formal.

8.3. Asymptotics. It seems likely that "most" moment-angle complexes are not formal, given a suitable way of making this statement precise. The following result is an example, strengthening Corollary 8.2.2.

Theorem 8.3.1. Let $a_{n}$ denote the number of n-point (labeled) triangulations of $S^{2}$, and let $b_{n}$ denote the number of such triangulations $K$ for which the momentangle manifold $\mathcal{Z}_{K}$ is formal. Then

$$
\lim _{n \rightarrow \infty} b_{n} / a_{n}=0 .
$$

Proof. Let $\mathcal{C}$ be the set of labeled triangulations of $S^{2}$. Then $\mathcal{C}$ is small and addable in the terminology of McDiarmid, Steger, and Welsh [35]. Their Theorem 4.1 then implies that the probability that a triangulation with $n$ vertices chosen uniformly at random contains a fixed, induced subgraph $H$ is bounded below by $1-e^{-c n}$, for a positive constant $c$, and for sufficiently large $n$.

Now choose $H$ to be one of the graphs in Figure 2. Using Theorem 6.1.1, we conclude that $\mathcal{Z}_{K}$ is not formal, for almost all $K \in \mathcal{C}$.

We do not know whether the analogous statement holds for isomorphism classes of triangulations of $S^{2}$.

8.4. Complex moment-angle manifolds. As mentioned in $\S 1.5$, work of López de Medrano-Verjovsky [39] and Meersseman [38] makes it possible to construct from combinatorial data compact, complex manifolds which are not algebraic, and even not Kähler or symplectic, except in very special cases.

If $K$ is an $n$-vertex polytopal triangulation of $S^{m}$ - that is, there exist $n$ points in $\mathbb{R}^{m+1}$ whose convex hull is isomorphic to $K$ as a simplicial complex-then from [8, Theorem 12.2], the moment-angle manifold $\mathcal{Z}_{K}$ admits a complex structure if its dimension $n+m+1$ is even. If $n+m+1$ is odd, then, by Remark 2.1.5, $\mathcal{Z}_{K, n+1}=\mathcal{Z}_{K} \times S^{1}$, and the theorem states that $\mathcal{Z}_{K, n+1}$ admits a complex structure.

For $m \geq 3$, not all triangulations of $S^{m}$ are polytopal. However, a classical theorem of Steinitz states that triangulations $K$ of $S^{2}$ are always polytopal. Then 
our constructions above of triangulations of $S^{2}$ for which $\mathcal{Z}_{K}$ is not formal apply to the corresponding LVM manifolds, too.

Corollary 8.4.1. There are infinitely many compact, complex manifolds arising from the López de Medrano-Verjovsky-Meersseman construction which are not formal.

In particular, by [15], none of these LVM manifolds is Kähler.

8.5. Indecomposable Massey products. In view of the theory developed so far, the question arises: are all Massey products in moment-angle complexes decomposable? The next example dispels this notion.

Example 8.5.1. Consider the 8-vertex simplicial complex $K$ with Stanley-Reisner ideal

$$
I_{K}=\left(x_{1} x_{2}, x_{1} x_{3} x_{4} x_{5}, x_{3} x_{4} x_{5} x_{6}, x_{3} x_{5} x_{6} x_{7}, x_{7} x_{8}\right) .
$$

(The construction of $K$ is inspired by an example of Backelin, reported in [2].) This simplicial complex has 1 three-face and 12 four-faces. The corresponding 12-dimensional moment-angle complex, $\mathcal{Z}_{K}$, has bigraded Betti numbers as listed below.

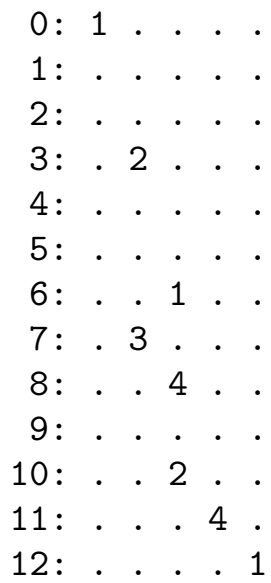

Claim. There is an indecomposable Massey product $\langle\alpha, \beta, \gamma\rangle$ in $H^{12}\left(\mathcal{Z}_{K}, \mathbb{k}\right)$, with $\alpha, \gamma \in H^{3}\left(\mathcal{Z}_{K}, \mathbb{k}\right)$ and $\beta \in H^{7}\left(\mathcal{Z}_{K}, \mathbb{k}\right)$.

Proof. Label the generators of $I_{K}$ as $e_{1}, \ldots, e_{5}$, and make the Taylor resolution on the exterior algebra generated by $e_{1}, \ldots, e_{5}$ (see e.g. [53]). A computation shows that $e_{1} e_{3}=-d\left(e_{1} e_{2} e_{3}\right)$, while $e_{3} e_{5}=-d\left(e_{3} e_{4} e_{5}\right)$. Thus, the Massey product $\left\langle e_{1}, e_{3}, e_{5}\right\rangle$ is defined. 
Note that, for each $p \neq 6$, either $b_{p}\left(\mathcal{Z}_{K}\right)=0$ or $b_{12-p}\left(\mathcal{Z}_{K}\right)=0$, and so $H^{p}$. $H^{12-p}=0$. Furthermore, $b_{6}\left(\mathcal{Z}_{K}\right)=1$, and so, by formula (25), we have $H^{6} \cdot H^{6}=$ 0 as well. Thus, $\left\langle e_{1}, e_{3}, e_{5}\right\rangle$ has zero indeterminacy, and is indecomposable.

Following Backelin [3] as in Remark 4.1.2, it is an exercise to show here that

$$
\sum_{p, q \geq 0} \operatorname{dim}_{\mathbb{k}} \operatorname{Tor}_{p, q}^{S / I}(\mathbb{k}, \mathbb{k}) s^{q} t^{p}=\frac{(1+s t)^{6}}{\left(1-s t-3 s^{6} t^{2}-s^{7} t^{3}\right)(1-s t)},
$$

which from (15), means

$$
h\left(U\left(\mathfrak{g}_{\mathcal{Z}}\right), t\right)=\left(\left(1-t-3 t^{6}-t^{7}\right)(1-t)(1+t)^{2}\right)^{-1} .
$$

The identity (16) makes it possible to compute the ranks $\phi_{r}$ of $\pi_{r}\left(\mathcal{Z}_{K}\right)$ recursively, and we find

$$
\begin{array}{l|llll}
r & 1234567891011 & 1213 \\
\hline \phi_{r} & 002000344 \quad 4 \quad 4 \quad 4 \quad 7
\end{array}
$$

The indecomposable Massey product above contributes a nonzero differential in the $E_{2}$ term of the Eilenberg-Moore spectral sequence, so the Lie algebra of primitives in $\operatorname{Ext}_{H^{*}\left(\mathcal{Z}_{K}, \mathbb{k}\right)}(\mathbb{k}, \mathbb{k})$ agrees with $\pi_{*}\left(\Omega \mathcal{Z}_{K}\right) \otimes \mathbb{k}$ up to but not including $\pi_{10}\left(\Omega \mathcal{Z}_{K}\right) \otimes \mathbb{k}$.

8.6. Bier spheres. We conclude this section with an example of a moment-angle manifold whose cohomology has indecomposable triple Massey products. Our approach uses a construction of triangulated spheres, due to Bier [7]. We briefly describe this construction here, and refer to [34], [32] for a complete discussion.

Let $K$ be proper simplicial subcomplex of the $n$-simplex. Then $K^{\star}$, its combinatorial Alexander dual, is defined to be the simplicial complex whose simplices are the complements in $[n]$ of the non-simplices of $K$. The Bier sphere associated to $K$ is the deleted join of $K$ with $K^{\star}$ :

$$
\operatorname{Bier}(K)=\left\{\sigma * \tau \in K * K^{\star} \mid \sigma \cap \tau=\emptyset\right\} .
$$

Remarkably, $\widehat{K}=\operatorname{Bier}(K)$ is a triangulation of $S^{n-2}$ on $2 n$ vertices: see [34], [32]. Consequently, the moment-angle complex $\mathcal{Z}_{\widehat{K}}$ is a manifold, of dimension $3 n-1$.

Example 8.6.1. If $K$ is the 4-gon, then $K^{\star}$ is the disjoint union of two edges, $\widehat{K}=\operatorname{Bier}(K)$ is the 8-vertex triangulation of $S^{2}$ from Figure 5 , and $\mathcal{Z}_{\widehat{K}}^{11}$ is the non-formal manifold from Example 8.1.1.

Example 8.6.2. Let $K$ be the 8-vertex simplicial complex from Example 8.5.1, and let $\widehat{K}=\operatorname{Bier}(K)$, a 16 -vertex triangulation of $S^{6}$. The manifold $\mathcal{Z}_{\widehat{K}}^{23}$ has 
Betti numbers

1, 0, 0, 10, 4, 12, 98, 130, 91, 233, 377, 268, 268, 377, 233, 91, 130, 98, 12, 4, 10, 0, 0, 1 .

Claim. The manifold $\mathcal{Z}_{\widehat{K}}$ carries an indecomposable Massey triple product $\langle\alpha, \beta, \gamma\rangle$ $\in H^{12}\left(\mathcal{Z}_{\widehat{K}}, \mathbb{k}\right)$.

Proof. Notice that $K$ is a full subcomplex of $\widehat{K}$, so we may apply the results of $\S 5.3$. Using Lemma 5.1.1, the Massey product in $C_{\mathrm{cW}}^{*}\left(\mathcal{Z}_{K}, \mathbb{k}\right)$ lifts through the split surjection $\mathcal{Z}_{f}^{*}: C_{\mathrm{cw}}^{*}\left(\mathcal{Z}_{\widehat{K}}, \mathbb{k}\right) \rightarrow C_{\mathrm{cw}}^{*}\left(\mathcal{Z}_{K}, \mathbb{k}\right)$ to a Massey product for $\mathcal{Z}_{\widehat{K}}$.

\section{Subspace arrangements}

Let $\mathcal{A}$ be an arrangement of linear subspaces in $\mathbb{C}^{n}$. The intersection lattice of the arrangement, $L(\mathcal{A})$, is the poset of all intersections among the subspaces in $\mathcal{A}$, ordered by reverse inclusion. The complement of the arrangement is simply the space $X(\mathcal{A})=\mathbb{C}^{n} \backslash \bigcup_{H \in \mathcal{A}} H$. A fundamental question in the subject is whether the homotopy type of the complement is completely determined by the combinatorial data, i.e., the intersection lattice and the codimensions of the subspaces.

9.1. Cohomology. In [25], Goresky and MacPherson gave a combinatorial formula for the Betti numbers of $X(\mathcal{A})$, one version of which we recall here:

$$
H^{i}(X(A), \mathbb{k})=\bigoplus_{S \in L(\mathcal{A})} \widetilde{H}_{2 \rho(S)-i-2}((\widehat{0}, S), \mathbb{k}),
$$

where $(\widehat{0}, S)$ denotes an open interval in the order complex of $L(\mathcal{A})$, and $\rho(S)$ is the complex codimension of $S$ in $\mathbb{C}^{n}$. Later on, the cohomology ring $H^{*}(X(\mathcal{A}), \mathbb{Z})$ was computed (also in purely combinatorial terms) by de Longueville and Schultz [33] and Deligne, Goresky, and MacPherson [14].

De Concini and Procesi observe in [13] that the mixed Hodge structure on the cohomology of a complex subspace complement of weight $2 r$ has type $(r, r)$, for each $r$. More precisely, Deligne, Goresky and MacPherson note [14, Exemple 1.14] that the summand of (43) labelled by $S$ is of type $(r, r)$, where $r=2 \rho(S)$.

9.2. Coordinate subspaces. Now let $K$ be a finite simplicial complex on vertex set $[n]$. Associated to $K$ there is a subspace arrangement $\mathcal{A}_{K}$ in $\mathbb{C}^{n}$, consisting of a coordinate subspace $H_{\sigma}=\left\{z \in \mathbb{C}^{n} \mid z_{i}=0\right.$ if $\left.i \notin \sigma\right\}$ for each non-empty simplex $\sigma \subseteq K$. It is readily seen that $X\left(\mathcal{A}_{K}\right)=\mathcal{Z}_{K}\left(\mathbb{C}, \mathbb{C}^{*}\right)$; thus, $X\left(\mathcal{A}_{K}\right)$ deform-retracts onto the moment-angle complex $\mathcal{Z}_{K}=\mathcal{Z}_{K}\left(D^{2}, S^{1}\right)$, cf. [9].

The subspaces in $\mathcal{A}_{K}$ are defined by equations $\left\{z_{i_{1}}=z_{i_{2}}=\cdots=z_{i_{k}}=0\right\}$, over index sets for which $x_{i_{1}} \cdots x_{i_{k}}$ is a generator of the Stanley-Reisner ideal $I=I_{K}$. Along the same lines, the intersection lattice $L(\mathcal{A})$ is isomorphic to both the 
lattice of least common multiples of the generators of $I$ (studied in [24]), and the opposite to the face lattice of the Alexander dual simplicial complex $K^{\star}$.

Using the second interpretation, Buchstaber and Panov observe in [9] that the Goresky-MacPherson formula (43) specializes for coordinate subspace arrangements to the classical Hochster formula (26), via Alexander duality. Indeed, for $p, q \geq 0$,

$$
\begin{aligned}
\operatorname{Tor}_{p-q, p+q}^{S}(S / I, \mathbb{k}) & \cong \bigoplus_{|\mathcal{I}|=p} \widetilde{H}^{q-1}\left(K_{\mathcal{I}}, \mathbb{k}\right) \\
& \cong \bigoplus_{|\mathcal{I}|=p} \widetilde{H}_{p-q-2}\left(\left(K_{\mathcal{I}}\right)^{\star}, \mathbb{k}\right) \\
& \cong \bigoplus_{\substack{\sigma \in K^{\star} \\
|\sigma|=n-p}} \widetilde{H}_{p-q-2}\left(\operatorname{link}_{K^{\star}}(\sigma), \mathbb{k}\right) .
\end{aligned}
$$

Since $H^{i}\left(X\left(\mathcal{A}_{K}\right), \mathbb{k}\right)=H^{i}\left(\mathcal{Z}_{K}, \mathbb{k}\right)=\operatorname{Tor}_{*, i}^{S}(S / I, \mathbb{k})$, the argument is completed by noting that the link of a simplex $\sigma$ in $K^{\star}$ is homeomorphic to its barycentric subdivision, the interval $(\widehat{0},[n]-\sigma)$ in $L(\mathcal{A})$. We refer to Eagon and Reiner $[18]$ for a related discussion.

The cohomology of the subspace complement $X\left(\mathcal{A}_{K}\right)$ inherits a bigrading from Tor. Let $\mathrm{gr}^{W}$ denote the grading associated to the weight filtration. Under the identification $H^{q}\left(X\left(\mathcal{A}_{K}\right), \mathbb{C}\right)=\operatorname{Tor}_{*, q}^{S}(S / I, \mathbb{C})$, then, keeping track of the bigrading above gives

$$
\operatorname{gr}_{r}^{W} H^{*}\left(X\left(\mathcal{A}_{K}\right), \mathbb{C}\right)=\bigoplus_{p} \operatorname{Tor}_{p, 2 r-p}^{S}(S / I, \mathbb{C}),
$$

for all $r \geq 0$.

9.3. The formality question. In [13] de Concini and Procesi gave a DGA model for the rational cohomology ring of a complex subspace arrangement $\mathcal{A}$. Using this model (as simplified by Yuzvinsky in [54]), Feichtner and Yuzvinsky [19] prove the following: If $L(\mathcal{A})$ is a geometric lattice, then $X(\mathcal{A})$ is a formal space.

Among arrangements with geometric intersection lattice, best understood are the "redundant" subspace arrangements, for which explicit computations of the homotopy Lie algebra can be done, under some assumptions; see [11, 47, 16]. However, it follows from [5] and the work above that the complement of a subspace arrangement need not be formal in general. Indeed, we have the following result.

Proposition 9.3.1. Suppose $K$ is a simplicial complex, containing as a full subcomplex one of the graphs listed in Figure 2. Then the complement of the coordinate subspace arrangement $\mathcal{A}_{K}$ is not formal. 


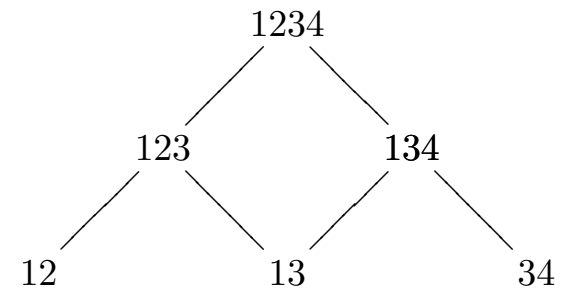

Figure 6. A non-geometric lattice

Proof. Recall the complement $X\left(\mathcal{A}_{K}\right)$ is homotopy equivalent to the momentangle complex $\mathcal{Z}_{K}$. The result thus follows from Theorem 6.1.1.

The simplest such example is obtained by letting $K$ be the simplicial complex of Figure 1. This is a simplicial complex on 6 vertices, with five minimal non-faces, and so $\mathcal{A}_{K}=\left\{H_{1}, \ldots, H_{5}\right\}$ where $H_{i}=\left\{z \in \mathbb{C}^{6} \mid z_{i}=z_{i+1}=0\right\}$. As expected, the intersection lattice of $\mathcal{A}_{K}$ is not geometric, either: the fragment of the lattice depicted in Figure 6 makes it clear why.

Acknowledgment. The first author would like to thank Srikanth Iyengar for helpful conversations regarding Golod maps and formality in commutative algebra. Both authors thank the referee for helpful suggestions that led to improvements in the presentation and content of the paper.

\section{REFERENCES}

1. Luchezar Avramov, Golod homomorphisms, Algebra, algebraic topology and their interactions, pp. 59-78, Lecture Notes in Math., vol. 1183, Springer, Berlin, 1986. MR 87m:13014

2. Luchezar Avramov, Infinite free resolutions, Six lectures on commutative algebra (Bellaterra, 1996), 1-118, Progr. Math., vol. 166, Birkhäuser, Basel, 1998. MR 99m:13022

3. Jürgen Backelin, Les anneaux locaux à relations monomiales ont des séries de Poincaré-Betti rationnelles, C. R. Acad. Sci. Paris Sér. I Math. 295 (1982), no. 11, 607-610. MR 85a:13008

4. Ivan Baskakov, Cohomology of $K$-powers of spaces and the combinatorics of simplicial divisions, Russian Math. Surveys 57 (2002), no. 5, 989-990 MR 2004b:55011

5. Ivan Baskakov, Triple Massey products in the cohomology of moment-angle complexes, Russian Math. Surveys 58 (2003), no. 5, 1039-1041. MR 2004j:55013

6. Ivan Baskakov, Victor Bukhshtaber and Taras Panov, Algebras of cellular cochains, and torus actions, Russian Math. Surveys 59 (2004), no. 3, 562-563. MR 2006b:57045

7. Thomas Bier, A remark on Alexander duality and the disjunct union, preprint, 1992.

8. Frédéric Bosio and Laurent Meersseman, Real quadrics in $\mathbb{C}^{n}$, complex manifolds and convex polytopes, Acta Mathematica 197 (2006), no. 1, 53-127.

9. Victor Bukhshtaber and Taras Panov, Actions of tori, combinatorial topology and homological algebra, Russian Math. Surveys 55 (2000), no. 5, 825-921. MR 2002a:57051

10. Ruth Charney and Michael W. Davis, Finite $K(\pi, 1)$ s for Artin groups, Prospects in topology (Princeton, NJ, 1994), 110-124, Ann. of Math. Stud., vol. 138, Princeton Univ. Press, Princeton, NJ, 1995. MR 97a:57001 
11. Daniel C. Cohen, Frederick R. Cohen and Miguel Xicoténcatl, Lie algebras associated to fiber-type arrangements, Intern. Math. Res. Not. 2003 (2003), no. 29, 1591-1621. MR 2005a:17019

12. Michael W. Davis and Tadeusz Januszkiewicz, Convex polytopes, Coxeter orbifolds and torus actions, Duke Math. J. 62 (1991), no. 2, 417-451. MR 92i:52012

13. Corrado De Concini and Claudio Procesi, Wonderful models of subspace arrangements, Selecta Math. (N.S.) 1 (1995), no. 3, 459-494. MR 97k:14013

14. Pierre Deligne, Mark Goresky, and Robert MacPherson, L'algèbre de cohomologie du complément, dans un espace affine, d'une famille finie de sous-espaces affines, Michigan Math. J. 48 (2000), 121-136. MR 2002b:14023

15. Pierre Deligne, Philip Griffiths, John Morgan, and Dennis Sullivan, Real homotopy theory of Kähler manifolds, Invent. Math. 29 (1975), 245-274. MR 52 \#3584

16. Graham Denham and Alexander Suciu, On the homotopy Lie algebra of an arrangement, Michigan Math. J. 54 (2006), no. 2, 319-340. MR 2252762

17. Alex Dranishnikov and Yuli Rudyak, Examples of non-formal closed $(k-1)$-connected manifolds of dimensions $\geq 4 k-1$, Proc. Amer. Math. Soc. 133 (2005), no. 5, 1557-1561. MR 2005h:55016

18. John Eagon and Victor Reiner, Resolutions of Stanley-Reisner rings and Alexander duality, J. Pure Appl. Algebra 130 (1998), no. 3, 265-275. MR 99h:13017

19. Eva Maria Feichtner and Sergey Yuzvinsky, Formality of the complements of subspace arrangements with geometric lattices, Zap. Nauchn. Sem. S.-Peterburg. Otdel. Mat. Inst. Steklov 326 (2005), 235-247. MR 2007a:55021

20. Yves Félix, Steven Halperin and Jean-Claude Thomas, Rational homotopy theory, Grad. Texts in Math., vol. 205. Springer-Verlag, New York, 2001. MR 2002d:55014

21. Marisa Fernández and Vicente Muñoz, On non-formal simply connected manifolds, Topology Appl. 135 (2004), no. 1-3, 111-117. MR 2004m:55016

22. Matthias Franz, On the integral cohomology of smooth toric varieties, preprint arXiv: math.AT/0308253

23. Ralph Fröberg, Koszul algebras, Advances in commutative ring theory (Fez, 1997), pp. 337350, Lecture Notes in Pure and Appl. Math., vol. 205, Dekker, New York, 1999. MR 2001i:16046

24. Vesselin Gasharov, Irena Peeva, and Volkmar Welker, The lcm-lattice in monomial resolutions, Math. Res. Lett. 6 (1999), no. 5-6, 521-532. MR 2001e:13018

25. Mark Goresky and Robert MacPherson, Stratified Morse theory, Ergeb. Math. Grenzgeb., vol. 14, Springer-Verlag, Berlin, 1988. MR 90d:57039

26. Dan Grayson and Michael Stillman, Macaulay 2: a software system for research in algebraic geometry; available at http://www.math.uiuc.edu/Macaulay2.

27. Jelena Grbić and Sthephen Theriault, Homotopy type of the complement of a coordinate subspace arrangement of codimension two, Russian Math. Surveys 59 (2004), no. 3, 12071209, MR 2005k:55023

28. Steven Halperin and James Stasheff, Obstructions to homotopy equivalences, Adv. Math. 32 (1979), no. 3, 233-279. MR 80j:55016

29. Melvin Hochster, Cohen-Macaulay rings, combinatorics, and simplicial complexes, in: Ring theory, II (Proc. Second Conf., Univ. Oklahoma, Norman, Okla., 1975), pp. 171-223, Lecture Notes in Pure and Appl. Math., vol. 26, Dekker, New York, 1977. MR 56 \#376

30. Ki Hang Kim and Fred Roush, Homology of certain algebras defined by graphs, J. Pure Appl. Algebra 17 (1980), no. 2, 179-186. MR 82e:05114b

31. Clas Löfwall, On the subalgebra generated by the one-dimensional elements in the Yoneda Ext-algebra, Algebra, algebraic topology and their interactions (Stockholm, 1983), Lecture Notes in Math., vol. 1183, Springer, Berlin, 1986, pp. 291-338. MR 88f:16030 
32. Mark de Longueville, Bier spheres and barycentric subdivision, J. Combin. Theory Ser. A 105 (2004), no. 2, 355-357. MR 2005d:52016

33. Mark de Longueville and Carsten Schultz, The cohomology rings of complements of subspace arrangements, Math. Ann. 319 (2001), no. 4, 625-646. MR 2002d:52009

34. Jiř̀ Matoušek, Using the Borsuk-Ulam theorem, Universitext, Springer-Verlag, Berlin, 2003. MR 2004i:55001

35. Colin McDiarmid, Angelika Steger and Dominic Welsh, Random planar graphs, J. Combin. Theory Ser. B 93 (2005), no. 2, 187-205. MR 2005k:05221

36. Dennis McGavran, Adjacent connected sums and torus actions, Trans. Amer. Math. Soc. 251 (1979), 235-254. MR 82c:57026

37. Brendan McKay, plantri, version 4.1, available at http://cs.anu.edu.au/ bdm/

38. Laurent Meersseman, A new geometric construction of compact complex manifolds in any dimension, Math. Ann. 317 (2000), no. 1, 79-115. MR 2001i:32029

39. Santiago López de Medrano and Alberto Verjovsky, A new family of complex, compact, non-symplectic manifolds, Bol. Soc. Brasil. Mat. 28 (1997), no. 2, 253-269. MR 98g:32047

40. John Meier and Leonard VanWyk, The Bieri-Neumann-Strebel invariants for graph groups, Proc. London Math. Soc. (3) 71 (1995), no. 2, 263-280. MR 96h:20093

41. Timothy Miller, On the formality of $(k-1)$-connected compact manifolds of dimension less than or equal to $4 k-2$, Illinois J. Math. 23 (1979), no. 2, 253-258. MR 80j:55017

42. John W. Milnor and John C. Moore, On the structure of Hopf algebras, Ann. of Math. (2) 81 (1965), 211-264. MR 30 \#4259

43. Dietrich Notbohm and Nigel Ray, On Davis-Januszkiewicz homotopy types. I. formality and rationalisation, Algebr. Geom. Topol. 5 (2005), 31-51. MR 2006a:55016

44. Taras Panov, Cohomology of face rings, and torus actions, preprint arXiv:math.AT/0506526

45. Taras Panov and Nigel Ray, Categorical aspects of toric topology, in preparation, see http://www.ma. man.ac.uk/ nige/cattabstract.pdf.

46. Taras Panov, Nigel Ray, and Rainer Vogt, Colimits, Stanley-Reisner algebras, and loop spaces, in: Categorical decomposition techniques in algebraic topology, Prog. Math., vol. 215, Birkhäuser, Basel, 2004, pp. 261-291. MR 2004k:55008

47. Stefan Papadima and Alexander Suciu, Homotopy Lie algebras, lower central series, and the Koszul property, Geometry \& Topology 8 (2004), 1079-1125. MR 2005g:55022

48. Stefan Papadima and Alexander Suciu, Algebraic invariants for right-angled Artin groups, Math. Ann. 334 (2006), no. 3, 533-555. MR 2006k:20078

49. Richard Stanley, Combinatorics and commutative algebra, Second edition, Progr. Math., vol. 41, Birkhäuser, Boston, MA, 1996. MR 98h:05001

50. James Stasheff, Rational Poincaré duality spaces, Illinois J. Math. 27 (1983), no. 1, 104-109. MR 85c:55012

51. Neil Strickland, Notes on toric spaces, preprint 1999, available at http://www.shef.ac.uk/ personal/n/nps/papers/

52. Dennis Sullivan, Infinitesimal computations in topology, Inst. Hautes Études Sci. Publ. Math. 47 (1977), 269-331. MR 58 \#31119

53. Sergey Yuzvinsky, Taylor and minimal resolutions of homogeneous polynomial ideals, Math. Res. Lett. 6 (1999), no. 5-6, 779-793. MR 2001i:13016

54. Sergey Yuzvinsky, Small rational model of subspace complement, Trans. Amer. Math. Soc. 354 (2002), no. 5, 1921-1945 MR 2003a:52030

Graham Denham

Department of Mathematics

University of Western Ontario

London ON N6A 5B7 
E-mail: gdenham@uwo.ca

http://www.math.uwo.ca/ ${ }^{\sim}$ gdenham

Alexander I. Suciu

Department of Mathematics

Northeastern University

Boston, MA 02115

E-mail: a.suciu@neu.edu

http://www.math.neu.edu/ ${ }^{\sim}$ suciu 